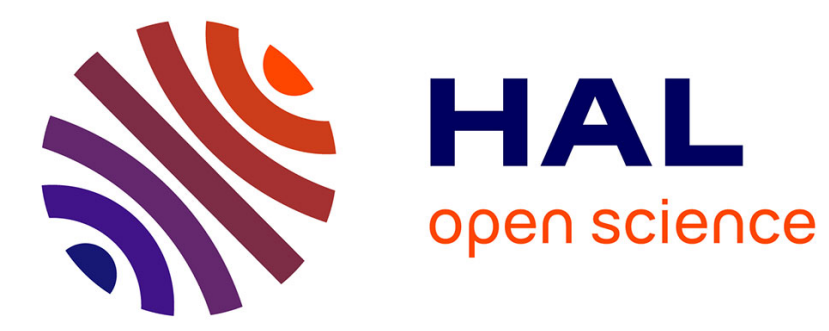

\title{
Spectral Overlap Optimization for DVB-T2 and LTE Coexistence
}

Hiba Bawab, Philippe Mary, Jean-François Hélard, Youssef Nasser, Oussama Bazzi

\section{- To cite this version:}

Hiba Bawab, Philippe Mary, Jean-François Hélard, Youssef Nasser, Oussama Bazzi. Spectral Overlap Optimization for DVB-T2 and LTE Coexistence. IEEE Transactions on Broadcasting, 2018, 64 (1), pp.70-84. 10.1109/TBC.2018.2795458 . hal-01770198

\section{HAL Id: hal-01770198}

\section{https://hal-univ-rennes1.archives-ouvertes.fr/hal-01770198}

Submitted on 7 Oct 2019

HAL is a multi-disciplinary open access archive for the deposit and dissemination of scientific research documents, whether they are published or not. The documents may come from teaching and research institutions in France or abroad, or from public or private research centers.
L'archive ouverte pluridisciplinaire HAL, est destinée au dépôt et à la diffusion de documents scientifiques de niveau recherche, publiés ou non, émanant des établissements d'enseignement et de recherche français ou étrangers, des laboratoires publics ou privés. 


\title{
Spectral Overlap Optimization for DVB-T2 and LTE Coexistence
}

\author{
Hiba Bawab, Philippe Mary, Member, IEEE, Jean-François Hélard, Senior \\ Member, IEEE Youssef Nasser, Senior Member, IEEE and Oussama Bazzi,
}

\begin{abstract}
In this paper, we analyze the spectral coexistence between the second generation terrestrial Digital Video Broadcasting (DVB-T2) and the Long Term Evolution (LTE) networks. In particular, the global spectral efficiency (SE) of both systems is investigated when a partial spectral overlap between DVB and LTE signals occurs. Our contribution lies in two-folds: i) the interfering signal variance in each network is derived analytically according to the frequency overlap while taking into account the difference between the characteristics of LTE and DVB networks, especially the OFDM subcarrier spacing and then the OFDM symbol duration. ii) the SE is derived with uniform and non uniform power allocation between overlapped and non overlapped subcarriers of the two networks. This derivation offers an analytical evaluation of the effect of the spectral overlap ratio variation and different power allocation scenarios and provides insightful results on the cooperation between the two networks.
\end{abstract}

\section{Index Terms}

DVB-T2, LTE, spectral overlap, power allocation, RF coexistence, interference cancellation (IC)

\section{INTRODUCTION}

Nowadays, the bandwidth is limited and the demand for high quality multimedia services is increasing. High data rate services are required, and spectral efficiency (SE) needs to be

Hiba Bawab is with Orange Labs Rennes, France (phone: +33-2-9912-4784, email: hiba.bawab@orange.com). The work has been performed when she was at INSA Rennes, France, and Lebanese University, Beirut, Lebanon

Philippe Mary is with INSA, IETR, CNRS UMR 6164, Rennes France (phone: +33-2-2323-8592, email: philippe.mary@insarennes.fr).

Jean-François Hélard is with INSA, IETR, CNRS UMR 6164, Rennes France.

Youssef Nasser is with American University of Beirut, Lebanon.

Oussama Bazzi is with Lebanese University, Beirut, Lebanon. 
maximized while the consumed power needs to be minimized. Hence, the maximization of SE and the minimization of the consumed power are today hot topics of interest in the wireless communication community. Moreover, when the universal TV broadcasting system has moved from analog to digital to satisfy the increasing demand on higher quality TV experience, a valuable part of the overcrowded frequency spectrum previously reserved for analog TV has been released. The Digital Video Broadcasting (DVB-T2) standard and the long term evolution (LTE) mobile services can both operate in a part of the digital dividend spectrum in the Ultra High Frequency (UHF) band. For instance, in France, according to the European commission recommendation, a part of the released spectrum $(790-862 \mathrm{MHz})$ is already used by LTE networks [1]. The third generation partnership project (3GPP) and DVB are becoming increasingly aware of the mutual benefits they can expect in terms of quality of service (QoS), profit sharing and power consumption in case of convergence for a common offer of services [2]. From the economical side, another noticeable advantage of the LTE-DVB cooperation has been studied in [3], [4] where the energy consumption of both systems has been shown to be always less than the total consumption in separate LTE and DVB networks. The decrease of energy is reflected in a decrease of operational fees and leads to an eco-friendly service network. In this context, this paper deals with the theoretical evaluation of the common SE of DVB-LTE systems when spectral overlap is authorized.

An important amount of works has been granted to the study of the spectral overlap coexistence of LTE-DVB systems. Among these studies, the acceptable level of interference, that both systems can support when they are located in the same or adjacent bands has been thoroughly studied with different conditions and criteria. Most of these works are based on simulations or experimental testbeds, i.e., the robustness of each system to the interference created by the other has been analyzed numerically.

For instance in [5] the authors have simulated a terminal DVB-T jammed by an LTE/WiMAX system (in downlink and uplink modes) located in adjacent channels and studied the protection ratio, defined as the ratio of the interference and desired signal powers, needed for an acceptable reception. LTE terminals in downlink and uplink, i.e. base stations (BS) or user equipment (UE), played the role of victims in [6], due to the interference created by a DVB transmission. Therein, the authors have evaluated the LTE throughput loss w.r.t. the adjacent channel interference ratio (ACIR).

In [7]-[10], the authors have built a set of experimental testbed to measure the impact of a 
coexistence between DVB-T2 and LTE in different scenarios, with either co-located or adjacent bands. The performance of DVB systems is evaluated using the quasi error free (QEF), the modulation error ratio (MER) or the received power criteria while the bit error rate (BER), the error vector magnitude (EVM) and channel quality indicator (CQI) are rather used for LTE. The authors have interestingly shown that the performance degradation of the DVB system is highly dependent on the bandwidth of the LTE signal and its RF level.

In [11]-[15], an experimental testbed dedicated to measure or to simulate the performance of DVB receivers under an interfering LTE signal has been performed. In particular, the authors have investigated the interference to carrier ratio when the LTE system is located in nearby UHF bands. They have quantified the protection distance for broadband system deployment when the latter is spectrally close to the DVB system.

Furthermore, some authors have even been interested in specific context such as the Australian LTE and DVB-T coexistence scenario in [16]-[18]. However, the studies are quite similar to the previous mentioned works and have dealt with the outage probability and/or the protection distance specification when some LTE devices operate in adjacent bands from DVB-T. Similarly, the Croatia context has been studied through the measurement of the throughput loss occurred in an LTE downlink communication overlapped by a DVB-T transmission in [19]. The authors in [20] have recently investigated potential coexistence issues between DVB and LTE networks in the 700 and $800 \mathrm{MHz}$ UHF bands by measuring interference protection ratios in laboratory conditions.

In [21], the authors have analyzed the adjacent channel interference effects of the LTE downlink signal on DVB-T home receivers. In [22], the authors have analyzed the case where indoor LTE-A femtocells coexist with DVB-T2 cells. In [23], filter-based interference mitigation techniques have been studied when LTE mobile system operating in Digital Dividend. In [24], the authors have investigated the impact of interfering mobile telecommunication services on the mobile TV services provided by LTE and DVB-T/T2 systems in the same frequency band. The studies in [21]-[24] have been based on measurements and simulations. Although all the above cited works are of interest, a signal-level description of mutual interference between both systems and their impact on each other performance have not been studied yet. In [25], [26], the mutual interference has been studied by deriving the ergodic capacity of both systems and evaluating its variation according to different system parameters. In these works, the ergodic capacity has been maximized and the optimal operating conditions have been given as a function of the ratio 
of the transmit powers of both systems. However, a rather simple interference model has been used in these works; if interesting insights on the effect of spectral overlap on SE have been given undoubtedly, the model does not capture what happens at the signal level. On the other hand, some studies have been based on SE to evaluate and optimize the spectral coexistence between two networks, e.g. [27]. However, this latter work has not been interested in giving a detailed expression of the mutual interference variance between the secondary and the primary signals. In our work, we aim at precisely describing the interference seen by the victim receiver, i.e. the primary and secondary receivers.

This paper aims at investigating how individual and global SE are varying when DVB and LTE bands overlap according to the spectral overlap ratio and signal parameters. The interference seen by a victim receiver, i.e. LTE or DVB, created by the interfering system, i.e. DVB or LTE respectively, is precisely described and the power of the interference is derived averaging over symbols, asynchronism and channel coefficients. The interference variance depends on both DVB-T2 and LTE characteristics and parameters, both systems being OFDM-based signals. From the interference power expressions, the individual and global average ergodic capacity are studied. Furthermore, the transmit power policy has an important impact on SE and hence, two transmit power scenarios and their impact on the global and individual SE are studied, i.e. uniform power allocation and interference aware power allocation. To the best of our knowledge, global SE of DVB and LTE systems under spectral overlap scenarios has not been derived analytically at signal level. This preliminary theoretical study could be a base for mobile operators and broadcasters aiming at complementing each other's network.

The remaining of this paper is organized as follows: Section II describes the system model. In Section III, the expression of the sampled signal on each sub-carrier is given taking into account the experienced interference. Then, the variance of interfering signals, i.e. DVB over LTE and LTE over DVB, are derived by averaging over symbols, asynchronism and channel coefficients. Section IV presents the expression of SE of the global system containing the DVB and LTE networks taking into account different power allocation scenarios. Section V deals with numerical results. Finally, conclusion is drawn in Section VI.

\section{SYSTEM MODEL}

The proposed model, described in Fig. 1, is composed of two communication systems: DVB and LTE networks. The DVB network is composed of one DVB transmitter (DT) and one DVB 


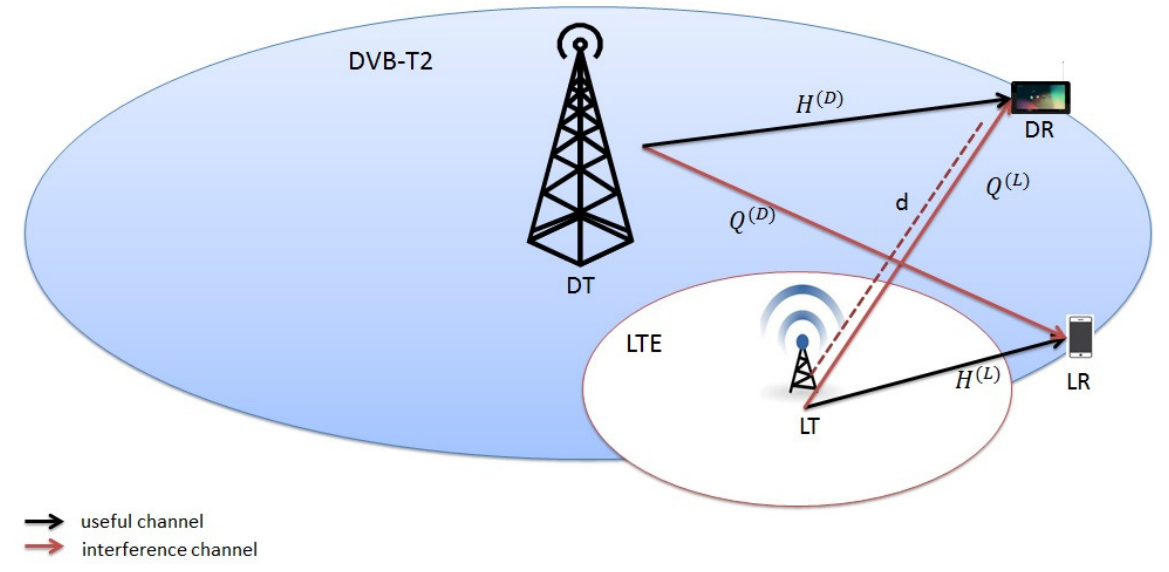

Fig. 1. System model: DVB-LTE coexistence

receiver (DR). The LTE system consists in a single cell of one LTE transmitter (LT) and one LTE receiver $(\mathrm{LR})^{1}$. Both systems, i.e. DVB and LTE, overlap spectrally as illustrated in Fig. 2.a. In this figure, $B^{(L)}$ and $B^{(D)}$ are the LTE and DVB bandwidths respectively, $\Delta f_{0}$ is the spectral overlap between $B^{(L)}$ and $B^{(D)}$ while $f_{c}$ and $f_{c}^{\prime}$ are their central frequencies such as $\Delta f=\left|f_{c}-f_{c}^{\prime}\right|$. Moreover, the spectral overlap ratio is defined by:

$$
\alpha=\frac{\Delta f_{0}}{B_{\min }}=\frac{1+\chi}{2 \chi}-\frac{\Delta f}{B_{\min }}
$$

where $\chi$ is defined by $\chi=\frac{B_{\min }}{B_{\max }}, B_{\min }=\min \left\{B^{(L)}, B^{(D)}\right\}$ and $B_{\max }=\max \left\{B^{(L)}, B^{(D)}\right\}$. Consequently, the interference arising between both bands depends on the frequency shift between them, i.e. the relative separation between the two networks bands.

Both DVB and LTE networks are OFDM-based systems but with different physical characteristics and different parameters such as different subcarrier spacing and sampling frequency. Let us define $T_{s}^{(L / D)}, T_{g}^{(L / D)}, N^{(L / D)}$ the useful part of OFDM symbol and cyclic prefix (CP) durations and the number of subcarriers of LTE and DVB systems respectively with $T^{(L / D)}=T_{s}^{(L / D)}+T_{g}^{(L / D)}$ the total symbol duration of OFDM symbols. Moreover, we consider $\xi=\frac{T^{(D)}}{T^{(L)}}=\frac{T_{s}^{(D)}}{T_{s}^{(L)}}=\frac{T_{g}^{(D)}}{T_{g}^{(L)}}>1$, such as $\xi \in \mathbb{Q}$.

\footnotetext{
${ }^{1}$ In real scenarios, multiple LTE cells should be considered but it is out of the scope of the paper and is left as further work. However, the adopted model can be seen as the case where two different LTE cells do not use the same frequency.
} 


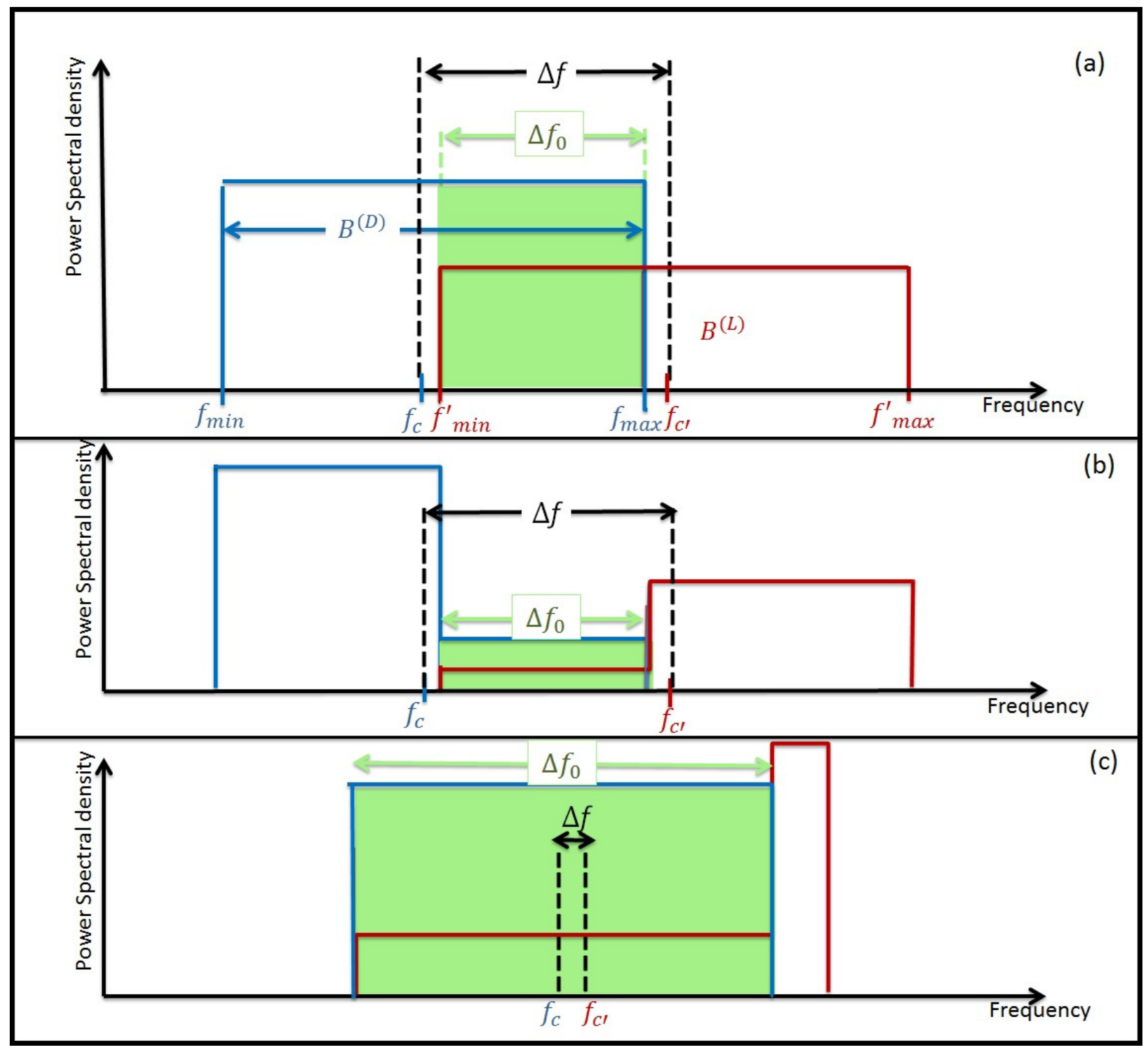

Fig. 2. Spectral overlap presentation

\section{INTERFERENCE MODEL}

The received signal in LR and DR depends on the interfering signal coming from the other network which has different characteristics. Hence, it can be obtained by writing the signal expressions of each system while taking into account the asynchronism and the different specifications. The asynchronism is simply due to the different time stamps of both systems. In this work, we do not consider signaling for synchronisation for both systems, but assume that perfect synchronisation of each system has been realized. This can be done in a traded cooperation phase, 
where both systems synchronise themselves without any interference before transmitting useful data in overlapping frequency bands.

\section{A. LTE received signal}

The OFDM signal waveform generated by LT is given by:

$$
s^{(L)}(t)=\frac{1}{\sqrt{T_{s}^{(L)}}} \sum_{l \in \mathbb{Z}} \sum_{k=0}^{N^{(L)}-1} X_{k}^{(L)}[l] e^{j 2 \pi \frac{k}{T_{s}^{(L)}} t} \Pi\left(\frac{t-l T^{(L)}}{T_{s}^{(L)}}\right)
$$

where $X_{k}^{(L)}[l]$ is the symbol of the $l$-th OFDM data block transmitted over the $k$-th LTE subcarrier. $\Pi(t)$ is the rectangular pulse shaping function defined by:

$$
\Pi(t)=\operatorname{rect}(t)= \begin{cases}1 & 0 \leq t \leq 1 \\ 0 & \text { otherwise }\end{cases}
$$

At the output of IFFT, a CP is added. Hence, we can write:

$$
s^{(L)}(t)=\frac{1}{\sqrt{T_{s}^{(L)}}} \sum_{l \in \mathbb{Z}} \sum_{k=0}^{N^{(L)}-1} X_{k}^{(L)}[l] e^{j 2 \pi \frac{k}{T_{s}^{(L)}} t} \Pi\left(\frac{t-l T^{(L)}+T_{g}^{(L)}}{T^{(L)}}\right)
$$

taking into account the multipath channel, the received signal at LR becomes:

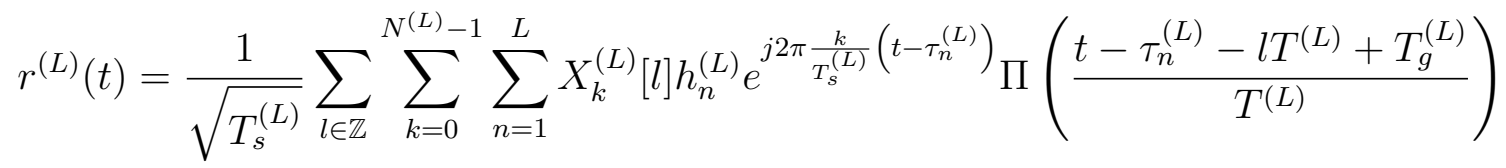

where $h_{n}^{(L)}$ and $\tau_{n}^{(L)}$ are respectively the LT-LR complex Gaussian channel coefficient and delay of the $n$-th path of the LTE channel which contains $L$ paths. The frequency response on the $k$-th subcarrier is given by $H_{k}^{(L)}=\sum_{n=1}^{L} h_{n}^{(L)} e^{-j 2 \pi \frac{k}{T_{s}^{(L)}} \tau_{n}}$. Hence, the received signal on the $p$-th subcarrier over the $m$-th OFDM data block is given by:

$$
\begin{aligned}
\tilde{X}_{p}^{(L)}[m] & =\int_{\mathbb{R}} r^{(L)}(t) \phi_{p, m}^{(L)}(t) d t \\
& =X_{p}^{(L)}[m] H_{p}^{(L)}[m]
\end{aligned}
$$

where $\phi_{p, m}^{(L)}(t)$ is the receiver waveform of the $m$-th OFDM block and the $p$-th subcarrier defined by $\phi_{p, m}^{(L)}(t)=\frac{1}{\sqrt{T_{s}^{(L)}}} e^{-j 2 \pi \frac{p}{T_{s}^{(L)}} t} \Pi\left(\frac{t-m T^{(L)}}{T_{s}^{(L)}}\right)$.

In order to compute the DVB interference power seen by the LTE receiver, we need to analyze the DVB signal seen by the victim receiver. Figure 3(a) illustrates the interference caused by DVB over the LTE signal. The duration of one DVB symbol encompasses several LTE symbols. Figure 3(b) shows that from LR point of view, the DVB signal is delayed with an unknown and 
asynchronism random variable $\theta^{(D)}$. Moreover, due to multipath propagation, multiple copies of a DVB symbol are received with delays $\tau_{n}^{(D)}, n \in \mathbb{N}$, as shown in Fig 3(c). By expressing the DVB signal received at the LTE receiver, the following result can be stated.

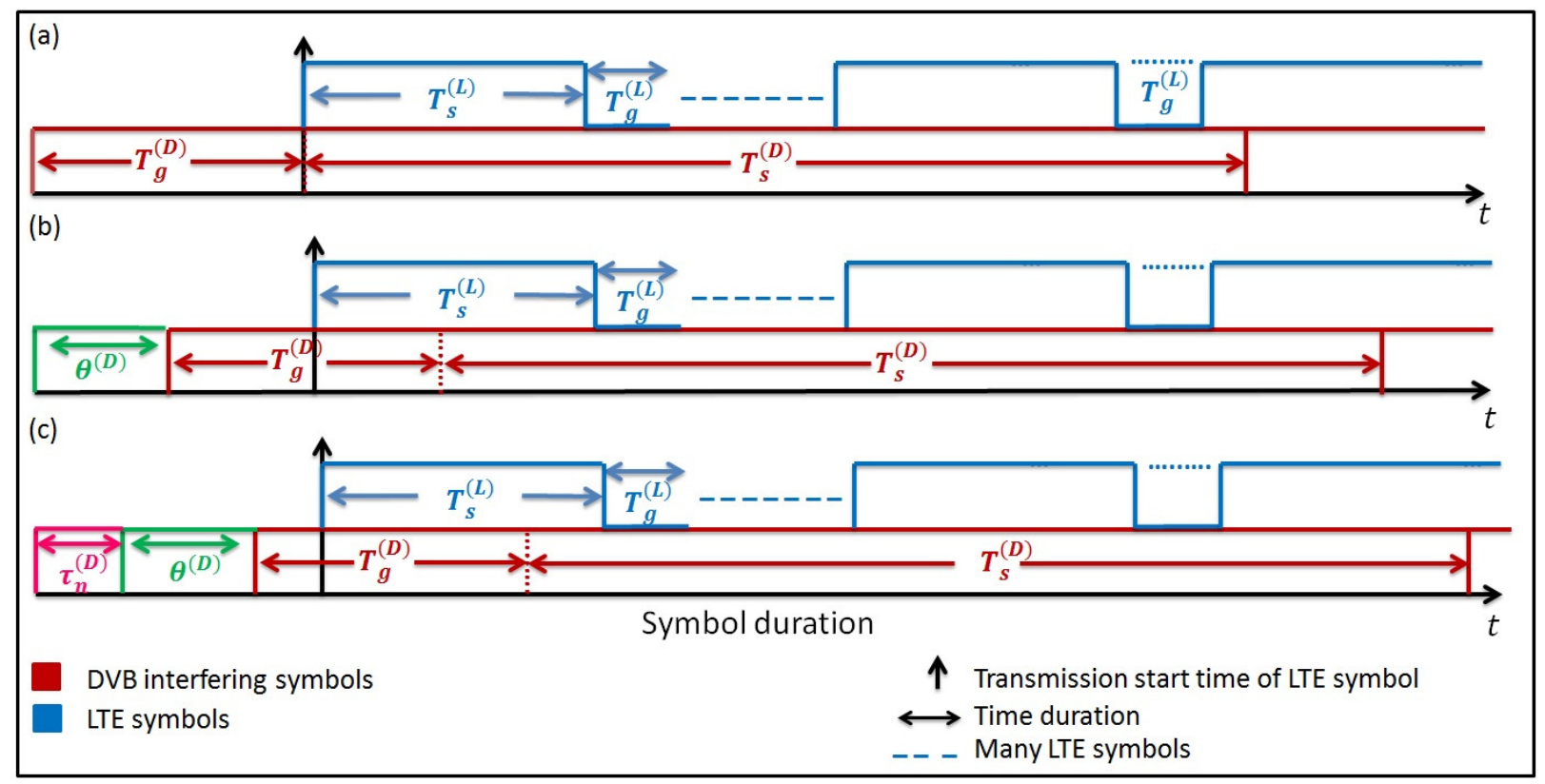

Fig. 3. Interfering DVB symbols over LTE symbol

Theorem III.1 (DVB interference variance over the LTE receiver). The DVB interference variance over the LTE receiver can be written as:

$$
V_{p}^{(D)}=\left(1-\frac{T_{s}^{(D)}}{\xi T^{(D)}}\right) \mathbb{E}_{x_{k^{\prime}}, q_{n^{\prime}}^{(D)}}^{(1)}\left[\left|I_{p}^{(D)}[m]\right|^{2}\right]+\mathbb{E}_{x_{k^{\prime}}, q_{n^{\prime}}^{(D)}, \theta^{(D)}}^{(2)}\left[\left|I_{p}^{(D)}[m]\right|^{2}\right]
$$

where $\theta^{(D)}$ is the unknown random asynchronism delay of DVB signal from LR point of view. $q_{n^{\prime}}^{(D)}$ and $\tau_{n^{\prime}}^{(D)}$ are respectively the DT-LR complex Gaussian distributed channel coefficient and delay of the $n^{\prime}$-th path of the DVB channel which contains $L^{\prime}$ paths. Its frequency response on the $k^{\prime}$-th subcarrier is defined as $Q_{k^{\prime}}^{(D)}=\sum_{n^{\prime}=1}^{L^{\prime}} q_{n^{\prime}}^{(D)} e^{-j 2 \pi \frac{k^{k}}{T_{s}^{(D)}} \tau_{n^{\prime}}} \cdot \mathbb{E}_{x_{k^{\prime}}, q_{n^{\prime}}^{(D)}}^{(1)}\left[\left|I_{p}^{(D)}[m]\right|^{2}\right]$ and $\mathbb{E}_{x_{k^{\prime}}, q_{n^{\prime}}^{(D)}, \theta^{(D)}}^{(2)}\left[\left|I_{p}^{(D)}[m]\right|^{2}\right]$ can be written as:

$$
\mathbb{E}_{X_{k^{\prime}}, q_{n^{\prime}}^{(D)}}^{(1)}\left[\left|I_{p}^{(D)}[m]\right|^{2}\right]=\frac{1}{\xi} \sum_{k^{\prime}=0}^{N^{(D)}-1}\left|\operatorname{sinc}\left(\pi\left(\frac{\Delta f^{(D)} T_{s}^{(D)}}{\xi}+\frac{k^{\prime}}{\xi}-p\right)\right)\right|^{2} \sum_{n^{\prime}=1}^{L^{\prime}} \Omega_{n^{\prime}}^{(D)}
$$




$$
\begin{aligned}
\mathbb{E}_{k_{k^{\prime}}, \theta^{(D), q_{n^{\prime}}(D)}}^{(2)}\left[\left|I_{p}^{(D)}[m]\right|^{2}\right]= & \frac{\xi}{\left(T_{s}^{(D)}\right)^{2} T_{s}^{(L)}} \sum_{k^{\prime}=0}^{N^{(D)}-1} \frac{1}{\pi^{2} A\left(k^{\prime}\right)^{2}} \sum_{n^{\prime}}^{L^{\prime}} \frac{\Omega_{n^{\prime}}}{2}\left\{2 \frac{T_{s}^{(D)}}{\xi}\right. \\
& +\frac{\sin \left(2 \pi A\left(k^{\prime}\right)\left(\frac{m T^{(D)}}{\xi}+\frac{T_{s}^{(D)}}{\xi}-B\left(n^{\prime}, \frac{T s^{(D)}}{\xi}\right)\right)\right)}{2 \pi A\left(k^{\prime}\right)} \\
& -\frac{\sin \left(2 \pi A\left(k^{\prime}\right)\left(\frac{m T^{(D)}}{\xi}+\frac{T_{s}^{(D)}}{\xi}-B\left(n^{\prime}, 0\right)\right)\right)}{2 \pi A\left(k^{\prime}\right)} \\
& \left.+\frac{\sin \left(2 \pi A\left(k^{\prime}\right)\left(\frac{m T^{(D)}}{\xi}-B\left(n^{\prime}, \frac{T s^{(D)}}{\xi}\right)\right)\right)}{2 \pi A\left(k^{\prime}\right)}\right\} \\
& \left.-\frac{\sin \left(2 \pi A\left(k^{\prime}\right)\left(\frac{m T^{(D)}}{\xi}-B\left(n^{\prime}, 0\right)\right)\right)}{2 \pi A\left(k^{\prime}\right)}\right\}
\end{aligned}
$$

where $\Omega_{n^{\prime}}^{(D)}=\mathbb{E}\left[\left|q_{n^{\prime}}^{(D)}\right|^{2}\right], A\left(k^{\prime}\right)=\Delta f^{(D)}+\frac{k^{\prime}}{T_{s}^{(D)}}-\frac{p}{T_{s}^{(L)}}$ and $B\left(n^{\prime}, \theta^{(D)}\right)=l^{\prime} T^{(D)}-T_{g}^{(D)}+\tau_{n^{\prime}}^{(D)}+\theta^{(D)}$ and $\Delta f^{(D)}$ is the frequency shift of DVB w.r.t. LTE.

The proof is given in Appendix A

\section{B. DVB received signal}

In the DVB network, the signal received by DR can be written as:

$$
r^{(D)}(t)=\frac{1}{\sqrt{T_{s}^{(D)}}} \sum_{l^{\prime} \in \mathbb{Z}} \sum_{k^{\prime}=0}^{N^{(D)}-1} \sum_{n^{\prime}=1}^{L^{\prime}} X_{k^{\prime}}^{(L)}\left[l^{\prime}\right] h_{n^{\prime}}^{(D)} e^{j 2 \pi \frac{k^{\prime}}{T_{s}^{(D)}}\left(t-\tau_{n^{\prime}}^{(D)}\right)} \Pi\left(\frac{t-\tau_{n^{\prime}}^{(D)}-l^{\prime} T^{(D)}+T_{g}^{(D)}}{T^{(D)}}\right)
$$

where $X_{k^{\prime}}^{(D)}\left[l^{\prime}\right]$ is the DVB complex symbol of the $l^{\prime}$-th OFDM data block over the $k^{\prime}$-th DVB subcarrier. $h_{n^{\prime}}^{(D)}$ is the DT-DR complex Gaussian distributed channel coefficient and its frequency response on the $k^{\prime}$-th subcarrier is given by $H_{k^{\prime}}^{(D)}=\sum_{n^{\prime}=1}^{L^{\prime}} h_{n^{\prime}}^{(D)} e^{-j 2 \pi \frac{k^{\prime}}{T_{s}^{(D)}} \tau_{n^{\prime}}}$.

The discrete signal is the projection of the received analog one on its waveform basis functions, i.e. OFDM waveform. Hence, the received signal at DR on the $p^{\prime}-$ th DVB subcarrier and over the $m^{\prime}$-th OFDM data symbol is given by:

$$
\tilde{X}_{p^{\prime}}^{(D)}\left[m^{\prime}\right]=\int_{\mathbb{R}} r^{(D)}(t) \phi_{p^{\prime}, m^{\prime}}^{(D)}(t) d t
$$

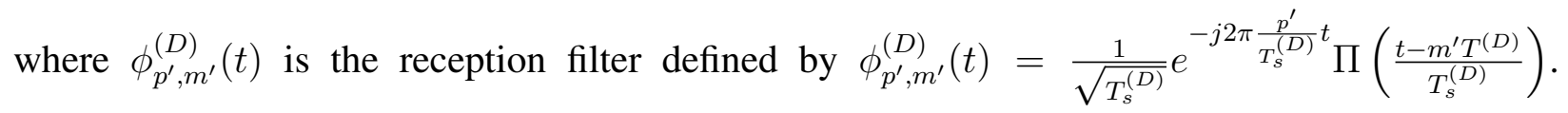
Applying the same calculation steps as in LTE case and considering that DT and DR are perfectly synchronized, the received DVB signal can be written as:

$$
\tilde{X}_{p^{\prime}}^{(D)}\left[m^{\prime}\right]=X_{p^{\prime}}^{(D)}\left[m^{\prime}\right] H_{p^{\prime}}^{(D)}\left[m^{\prime}\right]
$$




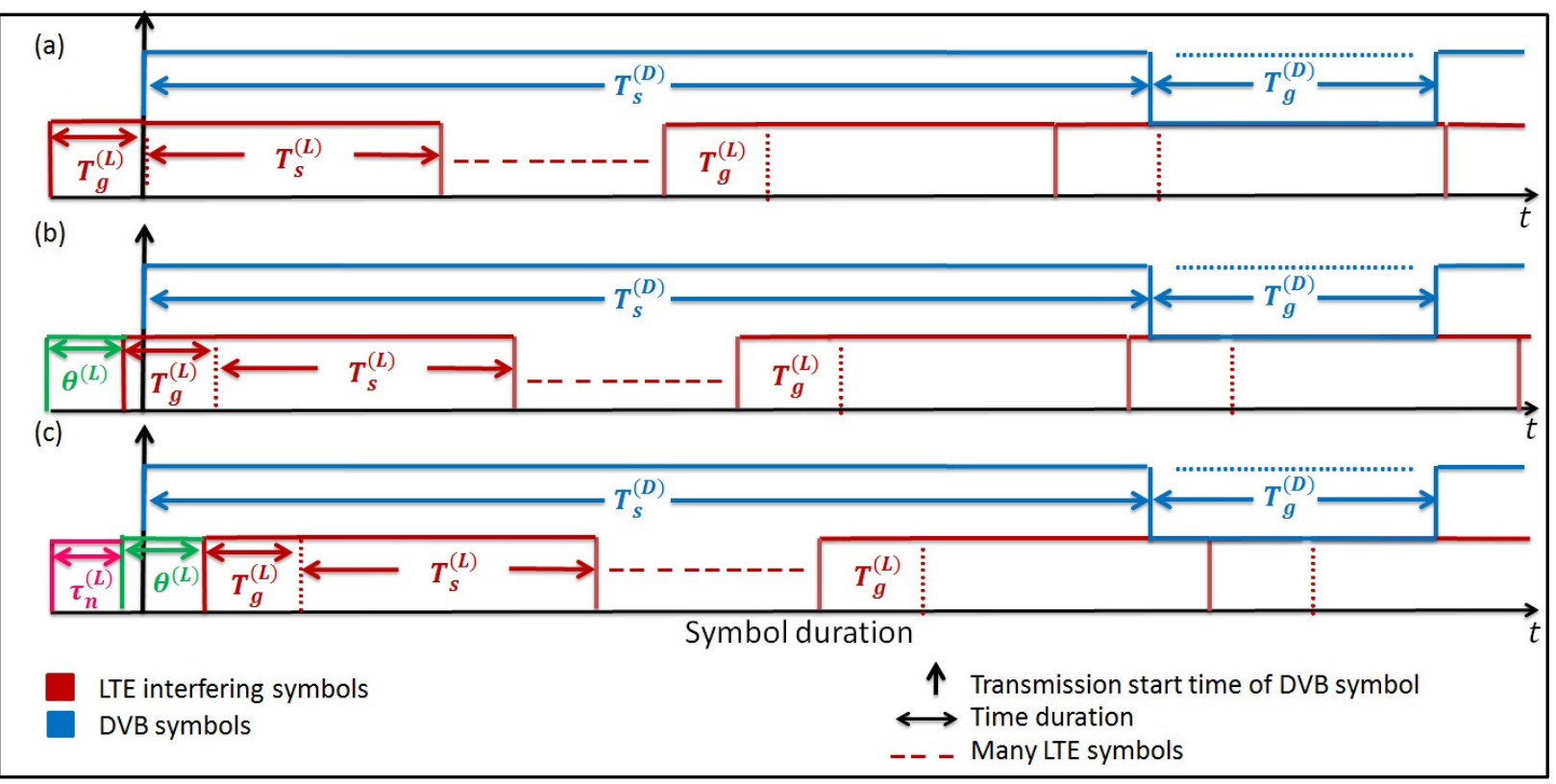

Fig. 4. Interfering LTE symbols over DVB symbol

Figure 4(a) shows the interference of several LTE symbols over one DVB symbol. From DR point of view, the LTE interfering signal is delayed with a random asynchronism, $\theta^{(L)}$, as shown in Fig. 4(b). Moreover, due to multipath propagation of LT-DR interference channel, multiple copies of LTE frames delayed by $\tau_{n}^{(L)}$ are received by DR as shown in Fig. 4(c). The power of the interference caused by LTE over the DVB receiver is given in the following theorem.

Theorem III.2 (LTE interference variance over the DVB receiver). The LTE interference variance over the DVB receiver can be written as:

$$
\begin{aligned}
V_{p^{\prime}}^{(L)} & =\mathbb{E}_{X_{k}, q_{n}^{(L)}, \theta^{(L)}}\left[\left|I_{p^{\prime}}^{(L)}\left[m^{\prime}\right]\right|^{2}\right] \\
& =\frac{1}{T_{s}^{(D)} T_{s}^{(L)} T^{(L)}} \sum_{k=0}^{N^{(L)}-1} \sum_{n=1}^{L} \Omega_{n}^{(L)}\left(\frac{1}{\pi^{2} C(k)^{2}}(T 1+T 2)+\left\lfloor\xi^{\prime}\right\rfloor\left(T^{(L)}\right)^{3} \operatorname{sinc}^{2}\left(\pi C(k) T^{(L)}\right)\right)
\end{aligned}
$$

where $q_{n}^{(L)}$ is the LT-DR complex Gaussian distributed channel coefficient where $Q_{k}^{(L)}$ is its frequency response on the $k$-th subcarrier defined as $Q_{k}^{(L)}=\sum_{n=1}^{L} q_{n}^{(L)} e^{-j 2 \pi \frac{k}{T_{s}^{(L)}} \tau_{n}^{(L)}} \cdot T 1$ and T2 are defined as:

$$
T 1=\frac{1}{2} T^{(L)}+\frac{\sin \left(2 \pi C(k)\left(m \xi T^{(L)}-G\left(n, T^{(L)}\right)\right)\right)-\sin \left(2 \pi C(k)\left(m \xi T^{(L)}-G(n, 0)\right)\right)}{4 \pi C(k)}
$$




$$
\begin{aligned}
T 2=\frac{1}{2} T^{(L)} & +\frac{\sin \left(2 \pi C(k)\left(\xi\left(m T^{(L)}+T_{s}^{(L)}\right)-\left\lfloor\xi^{\prime}\right\rfloor T^{(L)}-G\left(n, T^{(L)}\right)\right)\right)}{4 \pi C(k)} \\
& -\frac{\sin \left(2 \pi C(k)\left(\xi\left(m T^{(L)}+T_{s}^{(L)}\right)-\left\lfloor\xi^{\prime}\right\rfloor T^{(L)}-G(n, 0)\right)\right)}{4 \pi C(k)}
\end{aligned}
$$

where $C(k)=\Delta f^{(L)}+\frac{k}{T_{s}^{(L)}}-\frac{p^{\prime}}{T_{s}^{(D)}}, G\left(n, \theta^{(L)}\right)=l T^{(L)}-T_{g}^{(L)}+\tau_{n}^{(L)}+\theta^{(L)}$ and $\theta^{(L)}$ is the unknown and random asynchronism delay of LTE signal seen by DR.

The proof is given in Appendix $B$

\section{Power Allocation and Spectral EfFiciency}

In this section, individual and global SE are investigated under two power allocation scenarios: i) uniform power allocation, ii) interference aware power allocation with interference-cancellation ability.

\section{A. Uniform power allocation (UPA)}

The transmit power is assumed to be equally allocated to each subcarrier in each system whatever the frequency overlap ratio is. The ergodic data rates of LTE and DVB systems are given by:

$$
D^{(L / D)}=\sum_{p / p^{\prime}=0}^{N^{(L / D)}} \mathbb{E}\left[w_{p / p^{\prime}}^{(L / D)} \log _{2}\left(1+\operatorname{SINR}_{p / p^{\prime}}^{(L / D)}\right)\right]
$$

where $w_{p}^{(L)}$ and $w_{p^{\prime}}^{(D)}$ are the LTE and DVB subcarriers spacing respectively and the expectation is taken over the distribution of signal-to-interference plus noise ratio (SINR). SINRs experienced by LTE and DVB receivers are respectively given by:

$$
\operatorname{SINR}_{p / p^{\prime}}^{(L / D)}=\frac{\lambda^{(L / D)} l_{m / b}\left|H_{p / p^{\prime}}^{(L / D)}\right|^{2}}{\lambda^{(D / L)} l_{b m / m b} V_{p / p^{\prime}}^{(D / L)}+N_{0}^{(L / D)} w_{p / p^{\prime}}^{(L / D)}}
$$

where (17), interferences are considered as noise since they are only characterized by their variances. Expressions resulting from (16) are rather complex to obtain and in order to get practical insights with our theoretical findings, we focus on upper-bounds of previous expressions. With Jensen's inequality, we have:

$$
D^{(L / D)} \leq \sum_{p / p^{\prime}=0}^{N^{(L / D)}} w_{p / p^{\prime}}^{(L / D)} \log _{2}\left(1+\gamma_{p / p^{\prime}}^{(L / D)}\right)=\hat{D}^{(L / D)}
$$


where $\gamma_{p}^{(L)}, \gamma_{p^{\prime}}^{(D)}$ are the averaged SINR of LTE and DVB over the $p$-th and $p^{\prime}-$ th subcarrier respectively. They are given by:

$$
\gamma_{p / p^{\prime}}^{(L / D)}=\frac{\lambda^{(L / D)} l_{m / b} \bar{H}_{p / p^{\prime}}^{(L / D)}}{\lambda^{(D / L)} l_{b m / m b} V_{p / p^{\prime}}^{(D / L)}+N_{0}^{(L / D)} w_{p / p^{\prime}}^{(L / D)}}
$$

where $\bar{H}_{p}^{(L)}=\mathbb{E}\left[\left|H_{p}^{(L)}\right|^{2}\right]$ and $\bar{H}_{p^{\prime}}^{(D)}=\mathbb{E}\left[\left|H_{p^{\prime}}^{(D)}\right|^{2}\right]$. Moreover, $\lambda^{(L)}$ and $\lambda^{(D)}$ are respectively the transmit power per LTE and DVB subcarrier while $l_{m}, l_{b}, l_{b m}$ and $l_{m b}$ are the path loss attenuations of the LT-LR, DT-DR, DT-LR and LT-DR links respectively. Moreover, $N_{0}^{(L / D)}$ is the noise spectral density per LTE (DVB) subcarrier. The LTE (DVB) SE is given by $S^{(L / D)}=\frac{D^{(L / D)}}{B^{(L / D)}}$ where $B^{(L / D)}$ is the total bandwidth of LTE (DVB) system respectively. Hence, the global SE ${ }^{2}$ can be written as:

$$
S_{T}(\alpha)=\frac{1}{B_{T}(\alpha)}\left(\hat{D}^{(D)}(\alpha)+\hat{D}^{(L)}(\alpha)\right)
$$

where $B_{T}(\alpha)$ is the total used bandwidth given by

$$
B_{T}(\alpha)=B_{\min }(1-\alpha)+B_{\max }
$$

\section{B. Interference aware power allocation (IAPA)}

In the previous section, each system has been allocated with equal power between its subcarriers. However, based on the observation that, the more power assigned to good channels, i.e. without interference, the larger the achievable data rate, we assume the systems have the ability to allocate power differently on the interfering and non-interfering parts of the total bandwidth as illustrated in Fig. 2. For the system with the lowest bandwidth, i.e. $B_{\min }$, the total transmission power can be expressed as $P_{T}^{\min }=(1-\beta) P_{T}^{\min }+\beta P_{T}^{\min }$ where $P_{T}^{\min }$ is the total transmission power over the bandwidth $B_{\min }, \beta \in[0,1]$ is the interference aware power allocation (IAPA) parameter and $\beta P_{T}^{\min }$ is the power allocated to the interfering part and $(1-\beta) P_{T}^{\min }$ is the dedicated portion for the interference free part. Therefore, the transmission powers per subcarrier in the interfering and non-interfering parts are respectively $\lambda_{I}^{\min }=\frac{\beta P_{T}^{\min }}{K_{I}^{\min }}$ and $\lambda^{\min }=\frac{(1-\beta) P_{T}^{\min }}{K}$ with $K_{I}^{\min }=\alpha N^{\min }, K=(1-\alpha) N^{\min }$ and $N^{\min }$ the total number of subcarriers in $B_{\min }$. It is worth

\footnotetext{
${ }^{2}$ In this paper, the global SE is defined as the maximum sum rate of both systems provided to a given user normalized by the total occupied bandwidth.
} 
noting that the values of $K_{I}^{\mathrm{min}}$ and $K^{\mathrm{min}}$ depend on $\alpha$, which represents the interfering band ratio. The transmission power per one subcarrier can be re-written as:

$$
\begin{aligned}
\lambda_{I}^{\min } & =\frac{\beta}{\alpha} \lambda_{t}^{\min } \\
\lambda^{\min } & =\frac{1-\beta}{1-\alpha} \lambda_{t}^{\min }
\end{aligned}
$$

with $\lambda_{t}^{\min }=P_{T}^{\min } / N^{\min }$ is the transmission power value per subcarrier in case of uniform power allocation. On the other hand, for the system with the largest bandwidth, i.e. $B_{\max }$, the transmission powers per subcarrier in the interfering and non-interfering parts are respectively $\lambda_{I}^{\max }=\frac{\beta_{m} P_{T}^{\max }}{K_{I}^{\max }}$ and $\lambda^{\max }=\frac{\left(1-\beta_{m}\right) P_{T}^{\max }}{K}$ with $K_{I}^{\max }=\alpha_{m} N^{\max }, K^{\max }=\left(1-\alpha_{m}\right) N^{\max }$ and $\alpha_{m}=$ $\chi \alpha$. Moreover, $N^{\max }$ is the total number of subcarriers in $B_{\max }, P_{T}^{\max }$ is the total transmission power over the bandwidth $B_{\max }$. Similarly, $\beta_{m}$ is the fraction of power allocated to the interfering part of the system with the maximum bandwidth.

Two strategies for the power allocation, i.e. $\beta$ and $\beta_{m}$ can be envisaged: i) these two fractions are correlated to each other and when $\beta$ is fixed, the other is immediately obtained with $\beta_{m}=\chi \beta$ and ii) $\beta$ and $\beta_{m}$ are independent of each other. These cases will be thoroughly studied by simulations in Section V. Hence, the SINR expressions on the overlapped and non-overlapped LTE and DVB subcarriers are respectively given by:

$$
\begin{gathered}
\tilde{\gamma}_{p / p^{\prime}}^{(L / D)}=\frac{\lambda_{I}^{(L / D)} l_{m / b} \bar{H}_{p / p^{\prime}}^{(L / D)}}{l_{b m / m b}\left(\lambda_{I}^{(D / L)} V_{I}^{(D / L)}+\lambda^{(D / L)} V^{(D / L)}\right)+N_{0}^{(L / D)} w_{p / p^{\prime}}^{(L / D)}} \\
{\tilde{\gamma^{\prime}}}_{p / p^{\prime}}^{(L / D)}=\frac{\lambda^{(L / D)} l_{m / b} \bar{H}_{p / p^{\prime}}^{(L / D)}}{l_{b m / m b}\left(\lambda_{I}^{(D / L)} V_{I}^{(D / L)}+\lambda^{(D / L)} V^{(D / L)}\right)+N_{0}^{(L / D)} w_{p / p^{\prime}}^{(L / D)}}
\end{gathered}
$$

In this work, we have considered that DVB occupies a lower spectral range than LTE. Consequently, the rightmost subcarriers of DVB and the leftmost subcarriers of LTE are the more affected by the frequency overlap as seen in Fig. 2.a. This figure illustrates the uniform power allocation (UPA) scenario where the power spectral density (PSD) of each system is the same in the interfering and non-interfering parts. On the other hand, Fig. 2.b illustrates a case of IAPA scenario where the PSD of the non interfering part of both systems are greater than the one of the interfering part. Figure 2.c shows a full spectral overlap scenario where the DVB bandwidth is completely overlapped by LTE while this latter keeps a fraction of its bandwidth interference-free. In that case, only LTE is able to divide its power budget in the interfering 
and non-interfering parts. An interesting question is what is the optimal overlap ratio between bandwidths, i.e. $\alpha$, and the power allocation, i.e. $\beta$, which maximizes the global SE?

The received data rates of LTE and DVB networks in this allocation strategy are given by:

$$
\hat{D}^{\prime(L / D)}=\sum_{p / p^{\prime}=0}^{K_{I}^{(L / D)}-1} w_{p / p^{\prime}}^{(L / D)} \log _{2}\left(1+\tilde{\gamma}_{p / p^{\prime}}^{(L / D)}\right)+\sum_{p / p^{\prime}=K_{I}^{(L / D)}}^{N^{(L / D)}-1} w_{p / p^{\prime}}^{(L / D)} \log _{2}\left(1+{\tilde{\gamma^{\prime}}}_{p / p^{\prime}}^{(L / D)}\right)
$$

and the global SE w.r.t. $\alpha$ can be hence written as:

$$
S_{T}^{\prime}(\alpha)=\frac{1}{B_{T}(\alpha)}\left(\hat{D}^{(D)}\left(\alpha, \beta, \beta_{m}\right)+\hat{D}^{\prime(L)}\left(\alpha, \beta, \beta_{m}\right)\right)
$$

The next section consists in studying how individual and global SE behave when $\alpha$ and $\beta$ vary.

\section{IAPA with interference cancellation}

If the receivers have interference-cancellation abilities, the interference effect can be reduced and hence the overlap ratio could be enhanced while maintaining a high QoS [28], [29]. In particular, successive interference cancellation (SIC) receiver allows the interference coming from spectral overlapped signals to be reduced [30]-[35]. Indeed, let's assume that a system is able to remove a fraction $I_{R}$ of the interfering signal on each subcarrier at LTE and DVB receivers, therefore the interference term is multiplied by the term $\left|1-I_{R}\right|$ and hence the interference variance is multiplied by $\left|1-I_{R}\right|^{2}$. Consequently, we can prove that, in case of IAPA with interference cancellation, LTE and DVB SINR expressions can be written respectively as:

$$
\begin{aligned}
\tilde{\Gamma}_{p / p^{\prime}}^{(L / D)} & =\frac{\lambda_{I}^{(L / D)} l_{m / b} \bar{H}_{p / p^{\prime}}^{(L / D)}}{\left|1-I_{R}\right|^{2} l_{b m / m b}\left(\lambda_{I}^{(D / L)} V_{I}^{(D / L)}+\lambda^{(D / L)} V^{(D / L)}\right)+N_{0}^{(L / D)} w_{p / p^{\prime}}^{(L / D)}} \\
\Gamma_{p / p^{\prime}}^{(L / D)} & =\frac{\lambda^{(L / D)} l_{m / b} \bar{H}_{p / p^{\prime}}^{(L / D)}}{\left|1-I_{R}\right|^{2} l_{b m / m b}\left(\lambda_{I}^{(D / L)} V_{I}^{(D / L)}+\lambda^{(D / L)} V^{(D / L)}\right)+N_{0}^{(L / D)} w_{p / p^{\prime}}^{(L / D)}}
\end{aligned}
$$

Then, the achievable data rate and SE can be obtained by substituting the SINR expressions in (28), (29) in (26) and (27) respectively.

\section{Numerical Results}

Table I gives the parameters used throughout this section. The transmit power per subcarrier is obtained by dividing the maximum transmission power in each system by its total number of active subcarriers. Moreover, the LTE and DVB receivers are located respectively at the limits of 
TABLE I

GIVEN SYSTEM PARAMETERS

\begin{tabular}{|l|c|c|}
\hline Parameters & DVB-T2 & LTE-3GPP \\
\hline Bandwidth & $7.6 \mathrm{MHz}$ & $10 \mathrm{MHz}$ \\
\hline Subcarrier spacing & $1.116 \mathrm{kHz}$ & $15 \mathrm{kHz}$ \\
\hline Number of active subcarriers & 6817 & 601 \\
\hline Symbol period & $896 \mu s$ & $66.7 \mu s$ \\
\hline Guard interval time & $T_{s}^{(D)} / 4=224 \mu s$ & $T_{s}^{(L)} / 4=16.675 \mu s$ \\
\hline Cell radius & $100 \mathrm{~km}$ & $1 \mathrm{~km}$ \\
\hline Max transmission power & $10 \mathrm{~kW}$ & $50 \mathrm{~W}$ \\
\hline Transmit power/subcarrier & $1.395 \mathrm{~W}$ & $0.075 \mathrm{~W}$ \\
\hline Noise spectral density & $-165 \mathrm{dBm} / \mathrm{Hz}$ & $-165 \mathrm{dBm} / \mathrm{Hz}$ \\
\hline Path loss exponent & 2.5 & 3 \\
\hline
\end{tabular}

the LTE and DVB cells while the protection distance $d$ between the LTE transmitter and DVB receiver is a variable parameter in Figs. 5 and 6. However, $d=1000 \mathrm{~m}$ will be used in the other results throughout this section. Tables II and III present respectively the power delay profiles, i.e. the normalized average path power and path delays, of the Typical Urban (TUx) LTE 3GPP channel and the Typical Urban 6 (TU-6) DVB channel that are used respectively for the DT-LR and LT-DR channel interference models.

TABLE II

POWER DELAY PROFILE OF DVB-T2 TU-6 CHANNEL MODEL

\begin{tabular}{|l|c|c|c|c|c|c|}
\hline Tap & 1 & 2 & 3 & 4 & 5 & 6 \\
\hline Average power (dB) & -3 & 0 & -2 & -6 & -8 & -10 \\
\hline$\tau_{n^{\prime}}^{(D)} \mu s$ & 0 & 0.2 & 0.5 & 1.6 & 2.3 & 5 \\
\hline
\end{tabular}

TABLE III

POWER DELAY PROFILE OF 3GPP TUX CHANNEL MODEL

\begin{tabular}{|l|c|c|c|c|c|c|c|c|c|}
\hline Tap & 1 & 2 & 3 & 4 & 5 & 6 & 7 & 8 & 9 \\
\hline Average power (dB) & -5.7 & -7.6 & -10.1 & -10.2 & -10.2 & -11.5 & -13.4 & -16.3 & -16.9 \\
\hline$\tau_{n}^{(L)}(\mathbf{n s})$ & 0 & 217 & 512 & 514 & 517 & 674 & 882 & 1230 & 1287 \\
\hline
\end{tabular}

In Figures 5 and 6, the LTE and DVB data rates w.r.t. $\alpha$ are given respectively when UPA is 
applied. Both $D^{(L)}$ and $D^{(D)}$ decrease when the spectral overlap ratio $\alpha$ increases and moreover, LTE data rate is largely greater than DVB data rate. Obviously, $D^{(L)}$ is not affected by the distance LT-DR at contrary of DVB which is very sensitive to the protection distance between LT and DR, as expected. As illustrated in Fig. 6, when $d$ increases, the DVB data rate increases as it becomes less affected by the LTE interference. Figure 7 shows the global SE w.r.t $\alpha$ and labeled on $d$ when UPA is applied. The total SE increases when $d$ increases due to the increase of $D^{(D)}$. When $d$ is less than $1 \mathrm{~km}$, which means that DR is located inside the LTE cell, the total SE increases when the overlap ratio $\alpha$ increases despite that interference increases. This phenomena is marked by the red circle in Fig. 7. On the other hand, when $d$ becomes greater than $1 \mathrm{~km}$ which means that DR is located outside the LTE cell, the total SE increases when $\alpha$ increases to attain a maximal value and then decreases again, which highlights an optimal overlap ratio depending on the propagation model and system parameters.

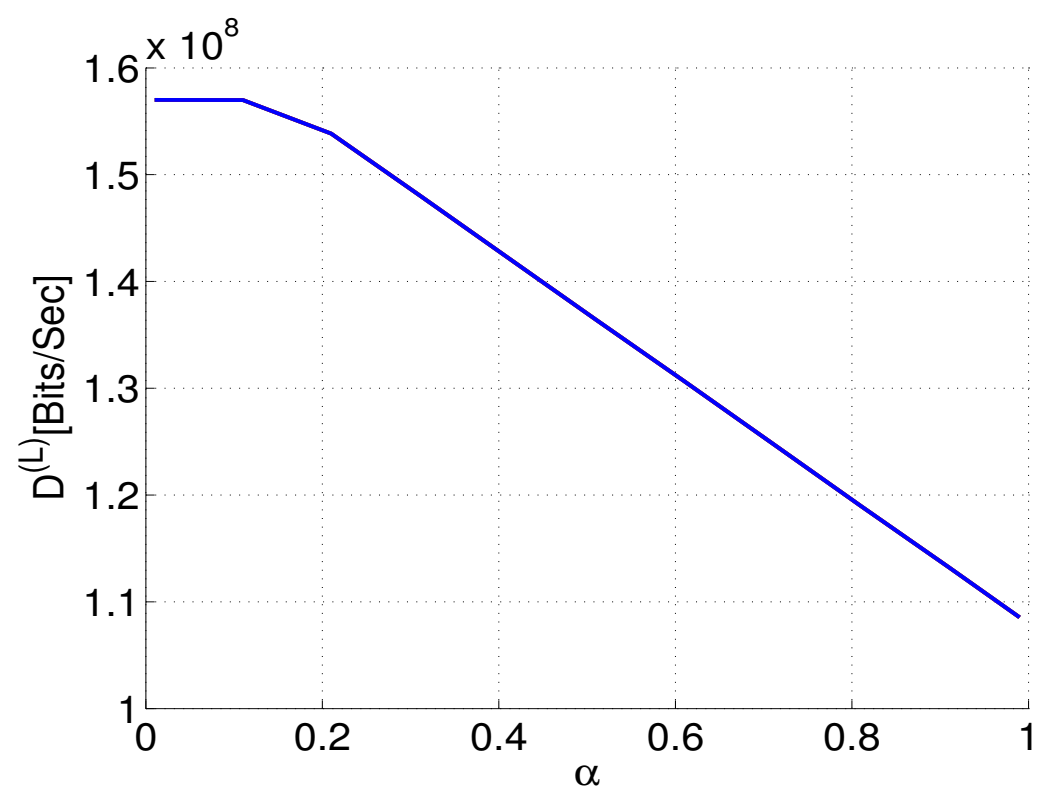

Fig. 5. LTE data rate in [Bits/sec] w.r.t. $\alpha$ when UPA is applied.

Figures 8, 9 and 10 draw the individual LTE, DVB data rates and global SE respectively w.r.t. $\alpha$ labeled on $I_{r}=\left|1-I_{R}\right|^{2}$ (the interference cancellation ratio) and $\beta$ when IAPA is applied. For that figures, $\beta_{m}=\chi \beta$ and hence the power allocated on the interfered LTE bandwidth part is linked to $\beta$. For a given overlap ratio $\alpha$, the LTE and DVB data rates improve when interference rejection mechanism is allowed; the lower $I_{r}$, i.e. a powerful interference canceler, the higher the improvement. However, the improvement gain is more appreciable on the DVB achievable rates 


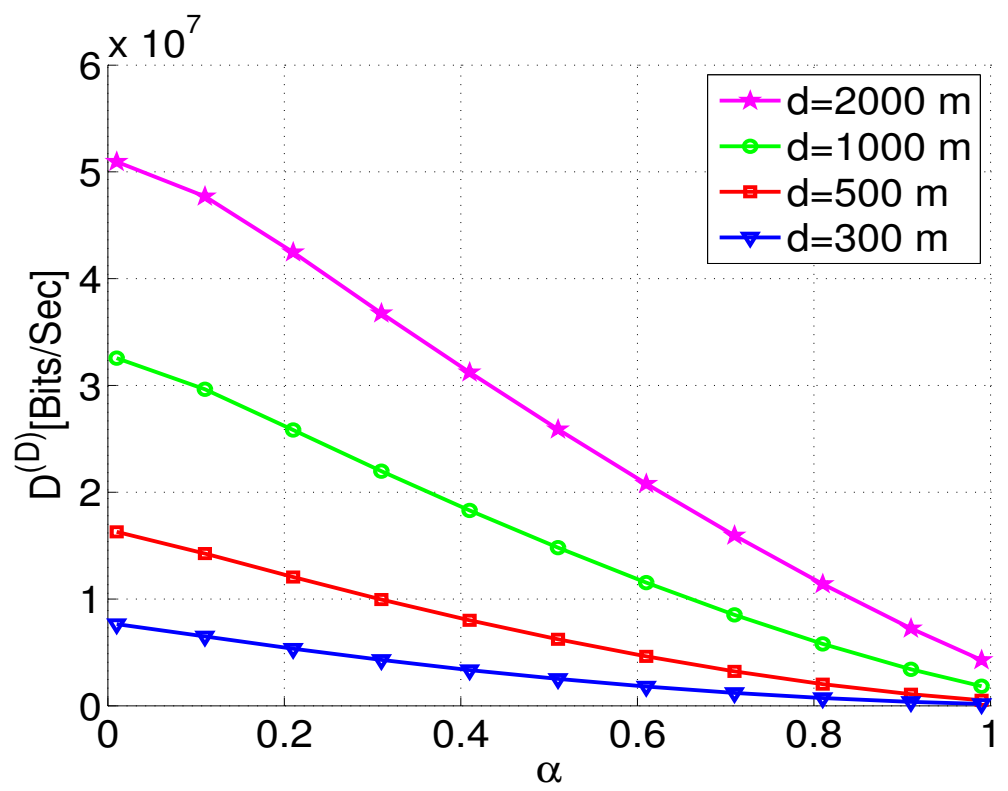

Fig. 6. DVB data rate in [Bits/sec] w.r.t. $\alpha$ and labeled on $\mathrm{d}=300,500,1000 \mathrm{~m}$ when UPA is applied

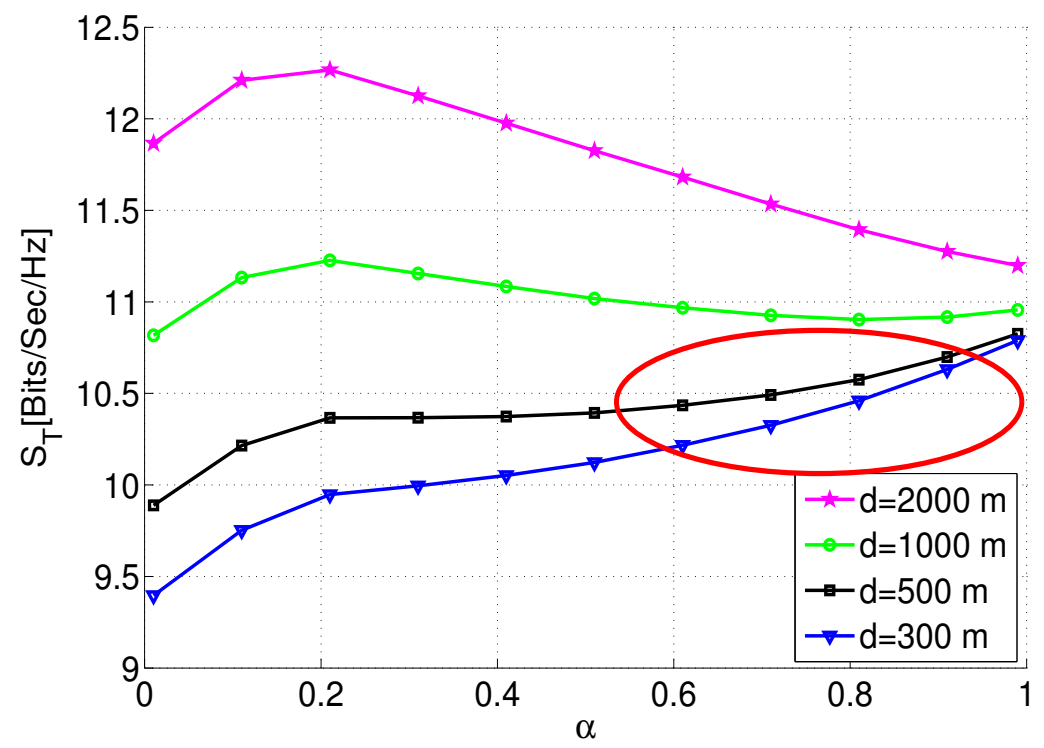

Fig. 7. Global spectral efficiency w.r.t. $\alpha$ and labeled on $\mathrm{d}=300,500,1000 \mathrm{~m}$ when UPA is applied.

because this system is more interference sensitive in our case. In addition, the data rates also improve when $\beta$ decreases, i.e. the portion of power allocated to the interfering part, for some given $I_{r}$ and $\alpha$. This illustrates the fact that IAPA and interference cancellation receiver can play a similar role in the improvement of SE when spectral overlap is authorized but these two 
techniques do not lead to the same technical solution. The former requires a power allocation at transmitters depending on the spectral overlap ratio, the latter implies more complex receivers to implement interference cancellation algorithms. Figure 8 shows the existence of an optimal overlap ratio around 0.1 maximizing the achievable LTE data rate. Figure 9 shows also a slightly optimal value of $\alpha$, also around 0.1 , but for $\beta=0.5,0.75$. In Fig. 10 the total SE roughly increases with $\alpha$ although both LTE and DVB data rates decreases for large overlap ratio. There are two main reasons for this behavior, i) when $\alpha$ increases the total used bandwidth decreases favoring an increase of SE as modeled in (21) and (20) and ii) even in case of full overlap, i.e. $\alpha=1$, the LTE data rate remains larger than the DVB one, due to the smaller LTE cell compared to the DVB cell size. We also remark when $\beta=0.25$ and whatever the values of $I_{r}$, the total SE is maximal for $\alpha \approx 0.9$. Hence, IAPA and interference rejection jointly applied may lead to a substantial gain in the global SE by favoring a large spectral overlap. However, this spectral overlap may be prejudicial to individual data rates as it can be inferred in Figs. 8 and 9 and then $\alpha$ should be chosen according to the data constraints that are imposed to each individual network.

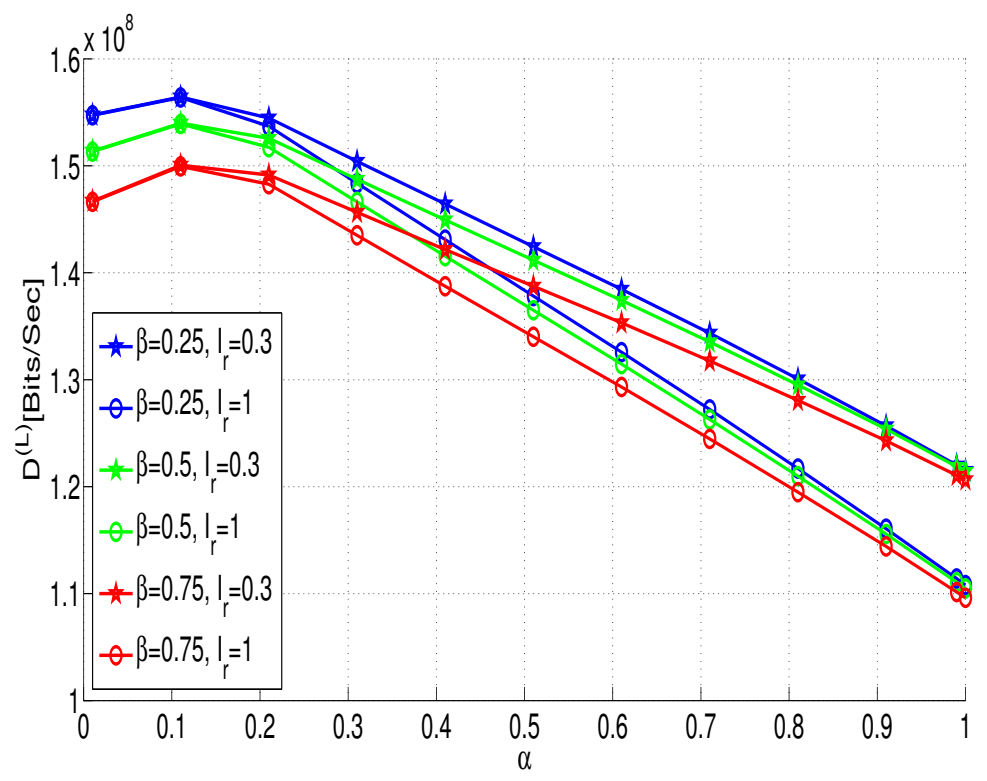

Fig. 8. LTE data rate w.r.t. $\alpha$ labeled on interference cancellation coefficient and IAPA parameter $\beta$ and $d=1000 \mathrm{~m}$.

As seen in the previous results, the individual and global data rate may be improved when IAPA is applied. Figures 11, 12 and 13 give more insights on how the individual and global data rates are varying according to $\alpha$ and $\beta$, with no interference cancellation ability. These 


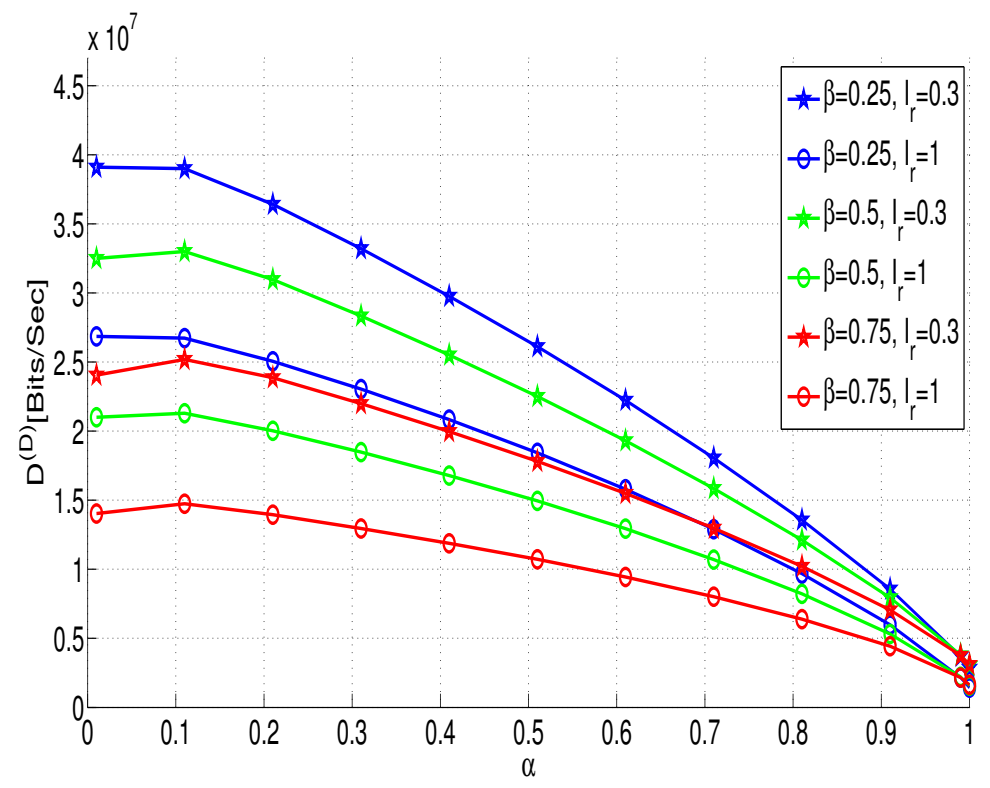

Fig. 9. DVB data rate w.r.t. $\alpha$ labeled on interference-cancellation coefficient and IAPA parameter $\beta$ and $d=1000 \mathrm{~m}$.

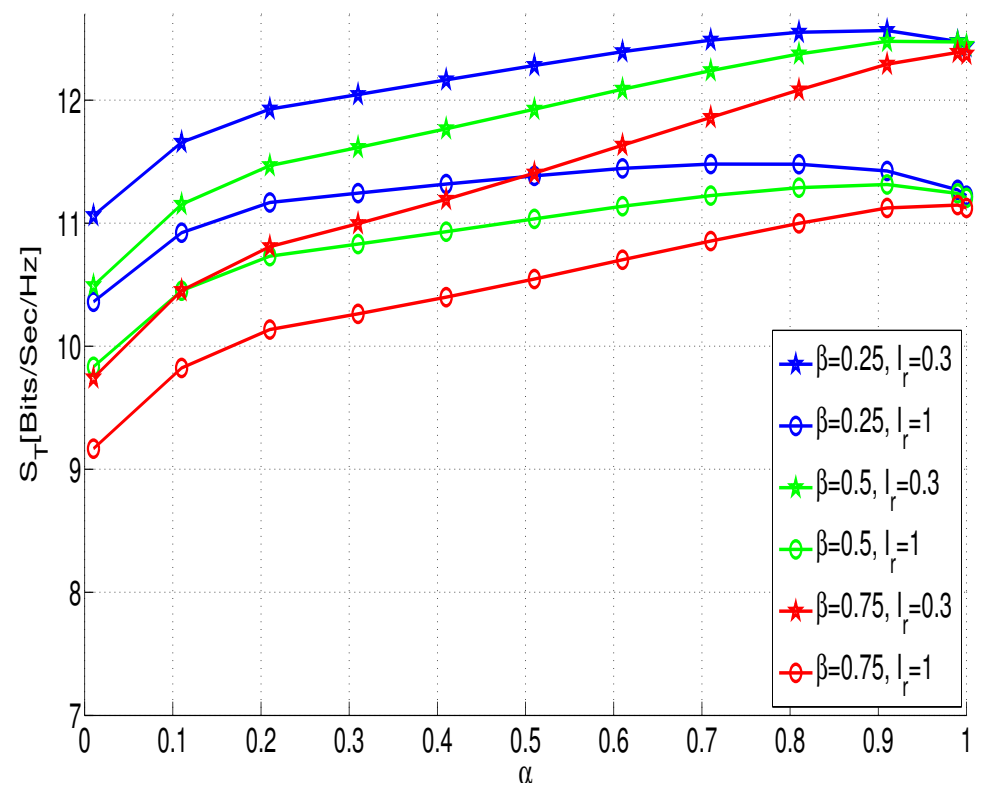

Fig. 10. Global spectral efficiency w.r.t. $\alpha$ labeled on interference-cancellation coefficient and IAPA coefficient $\beta$ and $d=1000$ m.

figures allow to choose the optimal values of $(\alpha, \beta)$ leading to the desired QoS in each network and/or the maximal global SE. As seen in Fig. 11, the DVB data rate increases when $\alpha$ and $\beta$ decreases. This means that, to increase the performance of the DVB network, one should decrease 
the spectral overlap ratio or increase the power allocation to the non-interfering part of the DVB bandwidth. On the other hand, the LTE data rate can have a maximal value for a combination of $\alpha$ and $\beta$ as observed in Fig. 12. But, in general perspective, it has an increasing behavior when $\alpha$ or $\beta$ decreases which means that the LTE network performance can be improved by decreasing the spectral overlap ratio or increasing the power allocation to the non-interfering part of the LTE bandwidth. Interestingly in Fig. 13, the total SE has a maximal value for a couple $(\alpha, \beta) \approx(0.9,0.1)$ implying a huge overlap ratio and almost all the power on the non-interfering part. The consequence of that strategy is that the system with the lowest bandwidth, i.e. DVB here, receives a degraded data rate. SE cannot be proved to be a convex or concave function of $\alpha$ and $\beta$, and hence the analytical study of the spectral overlap ratio might be very complex because of the really complicated dependency of data rates on $\alpha$ and $\beta$, cf Theorems III.1 and III.2.

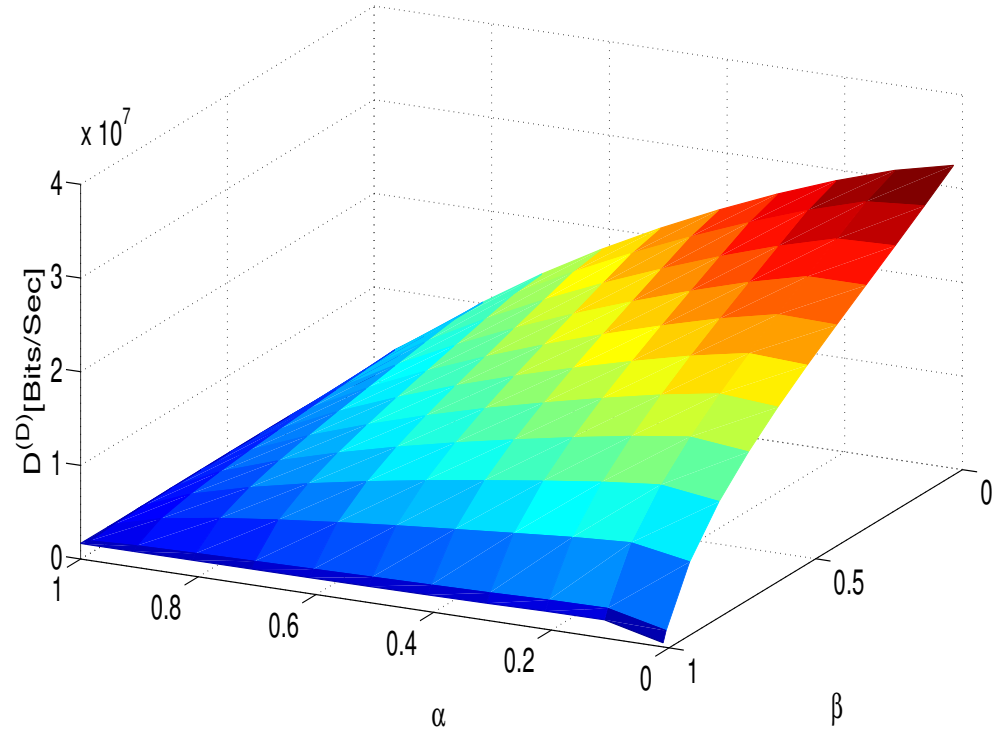

Fig. 11. DVB data rate w.r.t. $\alpha$ and $\beta$ when IAPA is applied, $d=1000 \mathrm{~m}$.

Figures 14, 15 and 16 show the individual and global data rates when $\beta$ and $\beta_{m}$ are kept free from each other and labeled on two values of $\alpha$, i.e. 0.3 and 0.7. From Figs. 14 and 15, it is clear that a lower spectral overlap, e.g. $\alpha=0.3$, is better for individual data rates, i.e. DVB and LTE, whatever the power allocation couple $\left(\beta, \beta_{m}\right)$ chosen. But the global SE can benefit from a higher spectral overlap, i.e. $\alpha=0.7$ depending on $\left(\beta, \beta_{m}\right)$, thanks to a lower occupied bandwidth and also because of the larger LTE data rate compared to the DVB rate. These results 


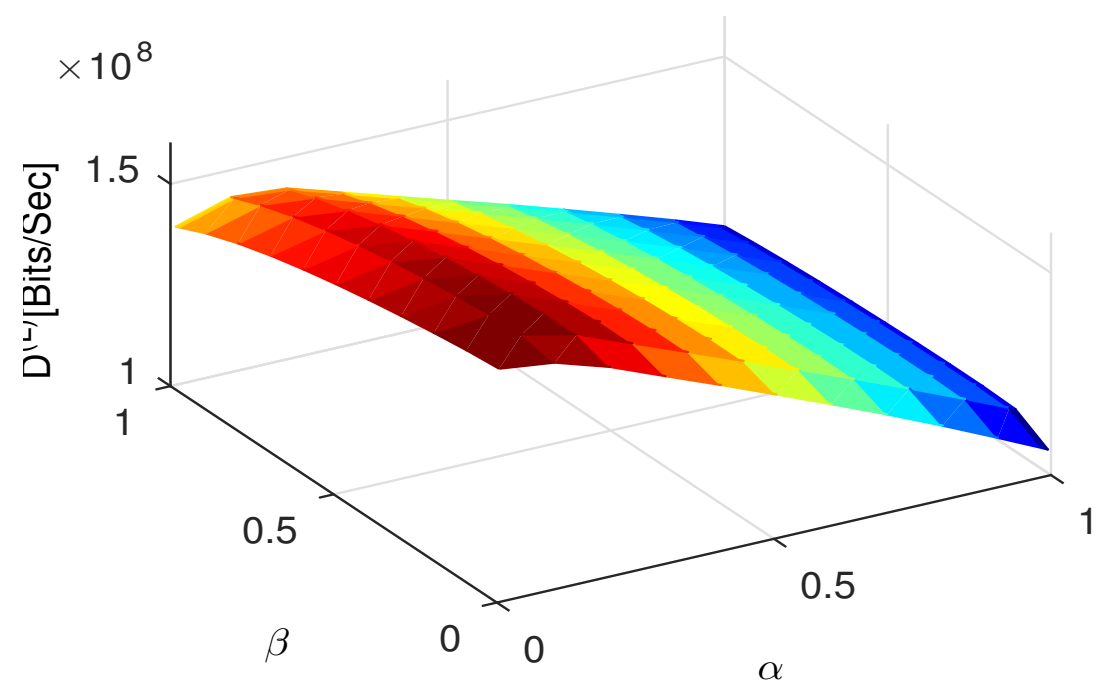

Fig. 12. LTE data rate w.r.t. $\alpha$ and $\beta$ when IAPA is applied, $d=1000 \mathrm{~m}$.

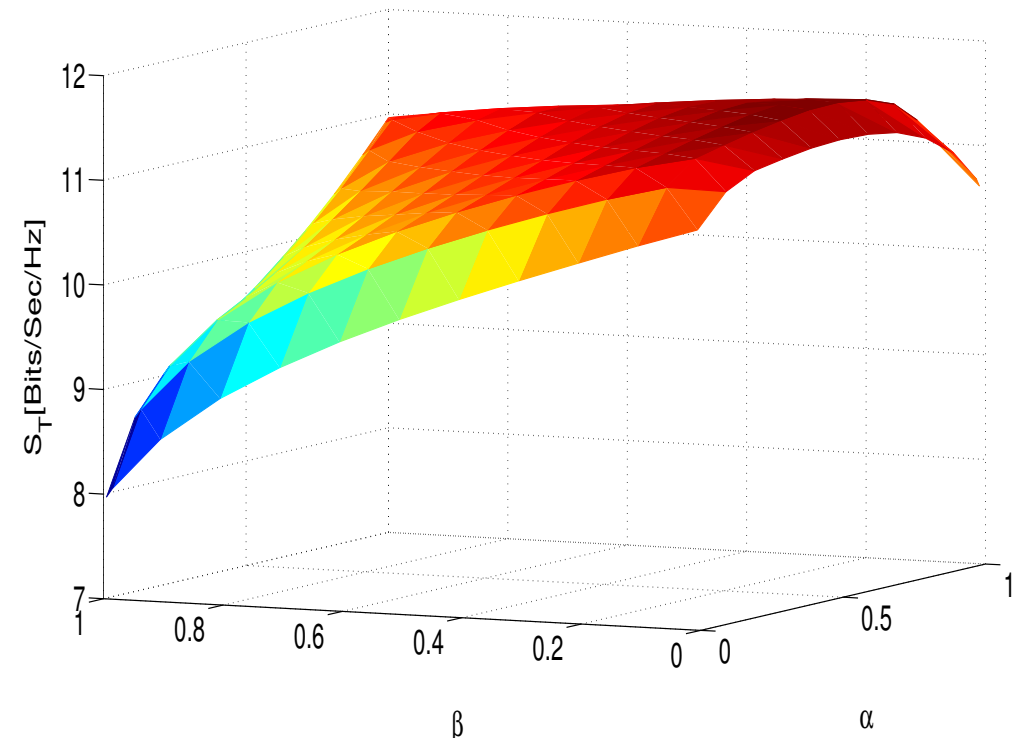

Fig. 13. Global spectral efficiency w.r.t. $\alpha$ and $\beta$ when IAPA is applied, $d=1000 \mathrm{~m}$.

enlighten the potentiality of spectral overlap between DVB and LTE to increase the global SE of the global system. 


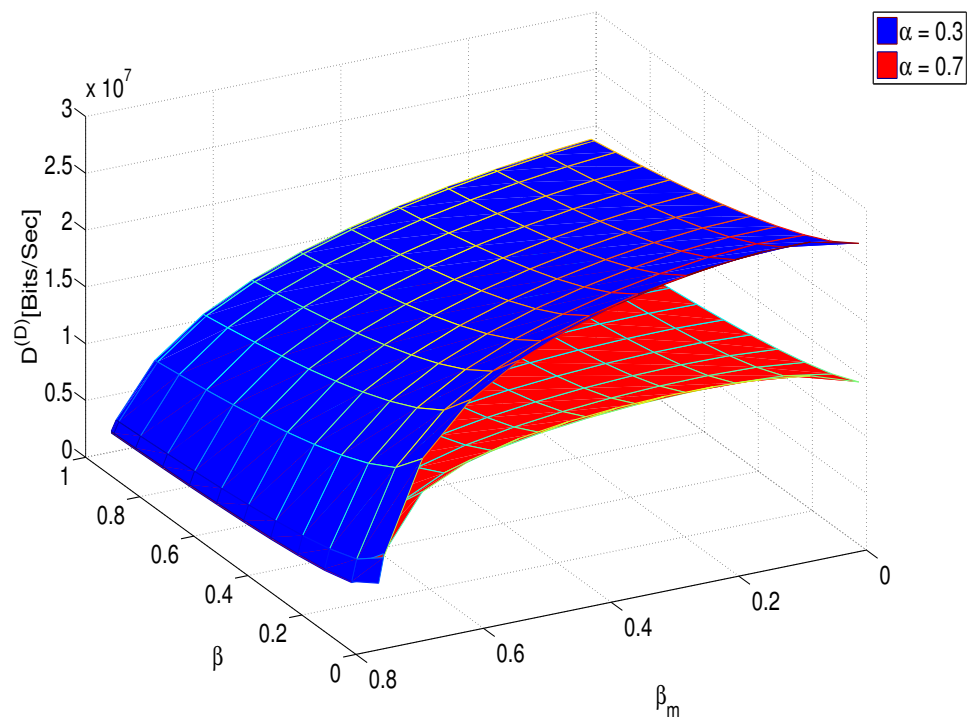

Fig. 14. DVB data rate w.r.t. $\beta$ and $\beta_{m}$ when IAPA is applied, $d=1000 \mathrm{~m}$, for $\alpha=0.3$ and $\alpha=0.7$.

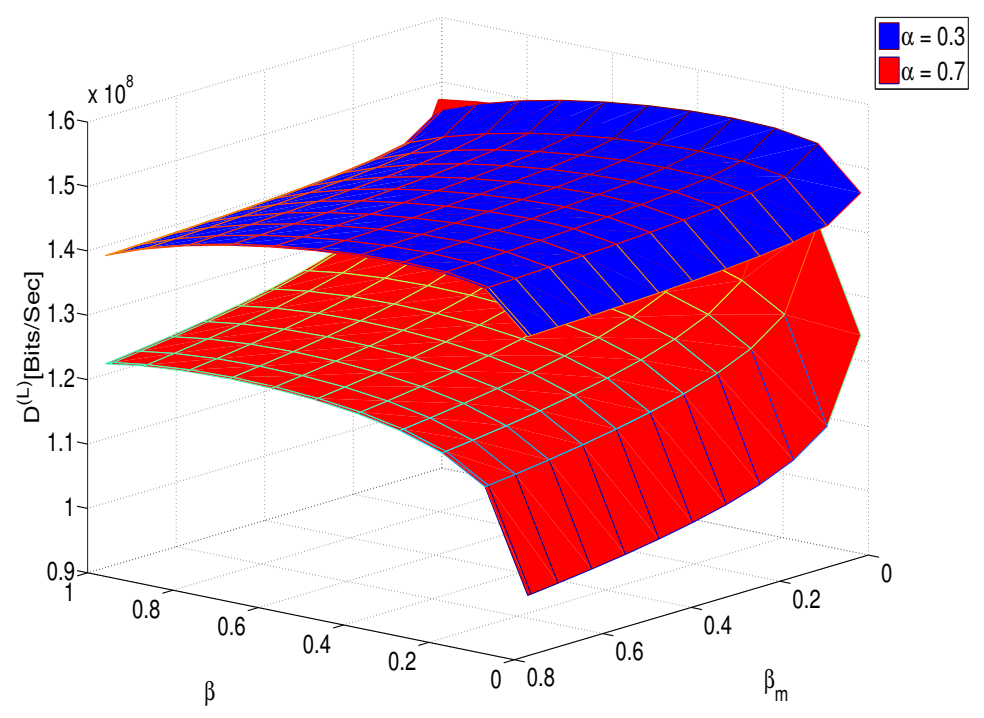

Fig. 15. LTE data rate w.r.t. $\beta$ and $\beta_{m}$ when IAPA is applied, $d=1000 \mathrm{~m}$, for $\alpha=0.3$ and $\alpha=0.7$.

\section{CONCLUSION}

In this paper, the impact on spectral efficiency of spectral overlap between LTE and DVB systems has been investigated. Our main contribution was to derive the variance of the interfering signal received on a given receiver, i.e. LTE or DVB, based on a continuous-time formulation. The 


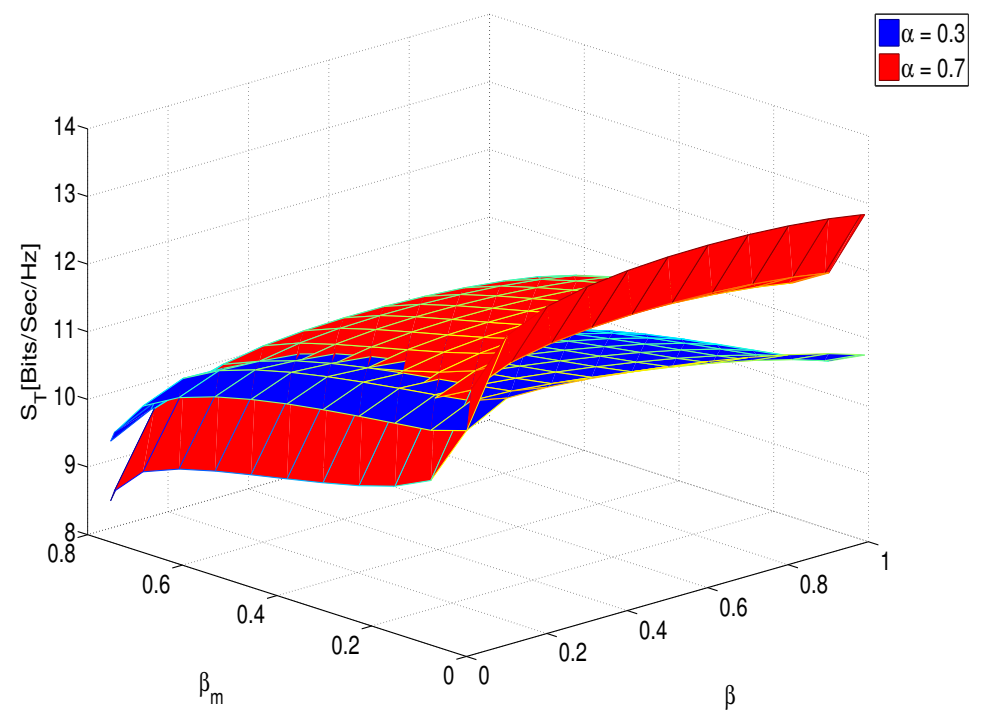

Fig. 16. Global spectral efficiency w.r.t. $\beta$ and $\beta_{m}$ when IAPA is applied, $d=1000 \mathrm{~m}$, for $\alpha=0.3$ and $\alpha=0.7$.

interference has been expressed w.r.t. LTE and DVB characteristics and random asynchronism between them. The interference signal has been shown to be asymmetric between LTE and DVB systems, mainly due to the differences between OFDM signal characteristics. The interference powers have been derived by successively averaging w.r.t. symbols, channel and asynchronism. Individual data rates and global SE have then been investigated according to the overlap ratio when the allocated power is the same on all subcarriers in a first time and when power can be allocated according to the overlap ratio. Moreover, in order to increase the achievable performance and find an optimal spectral overlap ratio, an advanced interference rejection ability has been assumed at the receivers. We have shown that uniform power allocation can be useful for the small values of spectral overlap ratio while the interference aware power allocation with interference-cancellation ability can be useful for higher values of spectral overlap ratio.

In this work, we have studied the optimal overlap ratio and power allocation between DVB and LTE through an accurate analytical model and numerical evaluation. However, a closedform expression of the optimal power strategy depending of the overlap ratio is still missing and is very challenging and left as further work. Moreover, the analysis with randomly deployed cellular BS in the area of the broadcast cell may be an interesting extension of this work. 


\section{APPENDIX A}

\section{ProOF OF THEOREM III. 1}

The transmitted OFDM DVB signal with $\mathrm{CP}$ is expressed as

$$
s^{(D)}(t)=\frac{1}{\sqrt{T_{s}^{(D)}}} \sum_{l^{\prime} \in \mathbb{Z}} \sum_{k^{\prime}=0}^{N^{(D)}-1} X_{k^{\prime}}^{(D)}\left[l^{\prime}\right] e^{j 2 \pi \frac{k^{\prime}}{T_{s}^{(D)}} t} \Pi\left(\frac{t-l^{\prime} T^{(D)}+T_{g}^{(D)}}{T^{(D)}}\right)
$$

The DVB interfering signal received by LTE before sampling can be expressed as:

$$
\begin{array}{r}
r^{(D)}(t)=\frac{e^{j 2 \pi \Delta f^{(D)} t}}{\sqrt{T_{s}^{(D)}}} \sum_{l^{\prime} \in \mathbb{Z}} \sum_{k^{\prime}=0}^{N^{(D)}-1} \sum_{n^{\prime}=1}^{L^{\prime}} X_{k^{\prime}}^{(D)}\left[l^{\prime}\right] e^{-j 2 \pi \frac{k^{\prime}}{T_{s}^{(D)}} \theta^{(D)} t} q_{n^{\prime}}^{(D)} e^{j 2 \pi \frac{k^{\prime}}{T_{s}^{(D)}}\left(t-\tau_{n^{\prime}}^{(D)}\right)} \\
\Pi\left(\frac{t-\tau_{n^{\prime}}^{(D)}-l^{\prime} T^{(D)}+T_{g}^{(D)}-\theta^{(D)}}{T^{(D)}}\right)
\end{array}
$$

LR decomposes the interfering DVB signal according to its own basis. Hence, the interference received on the $p$-th subcarrier and during the $m$-th LTE OFDM data block is given by:

$$
\begin{aligned}
I_{p}^{(D)}[m]= & \int_{\mathbb{R}} r^{(D)}(t) \phi_{p, m}^{(L)}(t) d t \\
= & \frac{1}{\sqrt{T_{s}^{(D)} T_{s}^{(L)}}} \sum_{k^{\prime}=0}^{N^{(D)}-1} e^{-j 2 \pi \frac{k^{\prime}}{T_{s}^{(D)}} \theta^{(D)} t} \sum_{l^{\prime} \in \mathbb{Z}} X_{k^{\prime}}^{(D)}\left[l^{\prime}\right] \sum_{n^{\prime}=1}^{L^{\prime}} q_{n^{\prime}}^{(D)} e^{-j 2 \pi \frac{k^{\prime}}{T_{s}^{(D)} \tau_{n^{\prime}}^{(D)}} \times} \\
& \int_{\mathbb{R}} e^{j 2 \pi\left(\Delta_{f}+\frac{k^{\prime}}{T_{s}^{(D)}}-\frac{p}{T_{s}^{(L)}}\right) t} \Pi\left(\frac{t-m T^{(L)}}{T_{s}^{(L)}}\right) \Pi\left(\frac{t-l^{\prime} T^{(D)}+T_{g}^{(D)}-\theta^{(D)}-\tau_{n^{\prime}}^{(D)}}{T^{(D)}}\right) d t
\end{aligned}
$$

The integration over $t$ depends on the term $\Pi\left(\frac{t-m T^{(L)}}{T_{s}^{(L)}}\right) \Pi\left(\frac{t-\tau_{n^{\prime}}^{(D)}-l^{\prime} T^{(D)}+T_{g}^{(D)}-\theta^{(D)}}{T^{(D)}}\right)$ where

$$
\begin{gathered}
\Pi\left(\frac{t-m T^{(L)}}{T_{s}^{(L)}}\right)=1 \quad \text { iff } \quad m T^{(L)} \leq t \leq m T^{(L)}+T_{s}^{(L)} \\
\Pi\left(\frac{t-\tau_{n^{\prime}}^{(D)}-l^{\prime} T^{(D)}+T_{g}^{(D)}-\theta^{(D)}}{T^{(D)}}\right)=1 \quad \text { iff } \quad l^{\prime} T^{(D)}+\tau_{n^{\prime}}^{(D)}-T_{g}^{(D)}+\theta^{(D)} \\
\leq t \leq\left(l^{\prime}+1\right) T^{(D)}+\tau_{n^{\prime}}^{(D)}-T_{g}^{(D)}+\theta^{(D)}
\end{gathered}
$$

Re-expressing the non-null conditions of (33) in terms of DVB characteristics, we have:

$$
\begin{aligned}
m \frac{T^{(D)}}{\xi} & \leq t \leq \frac{1}{\xi}\left(m T^{(D)}+T_{s}^{(D)}\right) \\
l^{\prime} T^{(D)}+\tau_{n^{\prime}}^{(D)}-T_{g}^{(D)}+\theta^{(D)} & \leq t \leq\left(l^{\prime}+1\right) T^{(D)}+\tau_{n^{\prime}}^{(D)}-T_{g}^{(D)}+\theta^{(D)}
\end{aligned}
$$


where $\theta^{(D)}$ is uniformly distributed over the entire OFDM symbol duration such that: $\theta^{(D)} \sim$ $\mathcal{U}\left(\left[0, T^{(D)}\right]\right)$. According to (35) and using Fig. 3, two cases exist:

case 1: One symbol interferes: $X_{k^{\prime}}^{(D)}\left[l^{\prime}-1\right]$ with the current LTE symbol $m . \forall n^{\prime}$, we have:

$$
\begin{aligned}
m \frac{T^{(D)}}{\xi} & >l^{\prime} T^{(D)}+\tau_{n^{\prime}}^{(D)}-T_{g}^{(D)}+\theta^{(D)} \\
\frac{1}{\xi}\left(m T^{(D)}+T_{s}^{(D)}\right) & \leq\left(l^{\prime}+1\right) T^{(D)}+\tau_{n^{\prime}}^{(D)}-T_{g}^{(D)}+\theta^{(D)}
\end{aligned}
$$

This condition is reached when:

$$
\left(\frac{m}{\xi}-l^{\prime}-1\right) T^{(D)}+\frac{T_{s}^{(D)}}{\xi}+T_{g}^{(D)}-\tau_{n^{\prime}}^{(D)} \leq \theta^{(D)} \leq\left(\frac{m}{\xi}-l^{\prime}\right) T^{(D)}+T_{g}^{(D)}-\tau_{n^{\prime}}^{(D)}
$$

case 2: Two symbols interfere: $X_{k^{\prime}}^{(D)}\left[l^{\prime}-1\right]$ and $X_{k^{\prime}}^{(D)}\left[l^{\prime}\right]$. This case is more complex than the first one and includes three sub-cases:

1) $\exists A$ such that $\forall n^{\prime}<A$, we have:

$$
\begin{aligned}
m \frac{T^{(D)}}{\xi} & >l^{\prime} T^{(D)}+\tau_{n^{\prime}}^{(D)}-T_{g}^{(D)}+\theta^{(D)} \\
\frac{1}{\xi}\left(m T^{(D)}+T_{s}^{(D)}\right) & \leq\left(l^{\prime}+1\right) T^{(D)}+\tau_{n^{\prime}}^{(D)}-T_{g}^{(D)}+\theta^{(D)}
\end{aligned}
$$

2) $\exists B$ such that $\forall n^{\prime}, A \leq n^{\prime}<B$ :

$$
\begin{aligned}
m \frac{T^{(D)}}{\xi} & \leq l^{\prime} T^{(D)}+\tau_{n^{\prime}}^{(D)}-T_{g}^{(D)}+\theta^{(D)} \\
\frac{1}{\xi}\left(m T^{(D)}+T_{s}^{(D)}\right) & \geq l^{\prime} T^{(D)}+\tau_{n^{\prime}}^{(D)}-T_{g}^{(D)}+\theta^{(D)}
\end{aligned}
$$

3) $\forall n^{\prime} \geq B$

$$
\begin{aligned}
m \frac{T^{(D)}}{\xi} & \geq\left(l^{\prime}-1\right) T^{(D)}+\tau_{n^{\prime}}^{(D)}-T_{g}^{(D)}+\theta^{(D)} \\
\frac{1}{\xi}\left(m T^{(D)}+T_{s}^{(D)}\right) & \leq l^{\prime} T^{(D)}+\tau_{n^{\prime}}^{(D)}-T_{g}^{(D)}+\theta^{(D)}
\end{aligned}
$$

In fact, this reformulation includes the first case of one interfering symbol where we have finally $A \geq L^{\prime}$. Rewriting these conditions of the three cases over $\theta^{(D)}$, we obtain:

1) $\exists A$ such that $\forall n^{\prime}<A$ and $A \leq L^{\prime}$, therefore one symbol interferes such as:

$$
\left(\frac{m}{\xi}-l^{\prime}-1\right) T^{(D)}+\frac{T_{s}^{(D)}}{\xi}+T_{g}^{(D)}-\tau_{n^{\prime}}^{(D)} \leq \theta^{(D)} \leq\left(\frac{m}{\xi}-l^{\prime}\right) T^{(D)}+T_{g}^{(D)}-\tau_{n^{\prime}}^{(D)}
$$

2) $\exists B$ such that $\forall n^{\prime} \in\left[A, B\left[\right.\right.$ and $B \leq L^{\prime}$ :

$$
\left(\frac{m}{\xi}-l^{\prime}\right) T^{(D)}+T_{g}^{(D)}-\tau_{n^{\prime}}^{(D)} \leq \theta^{(D)} \leq\left(\frac{m}{\xi}-l^{\prime}\right) T^{(D)}+\frac{T_{s}^{(D)}}{\xi}+T_{g}^{(D)}-\tau_{n^{\prime}}^{(D)}
$$


3) $\forall n^{\prime} \geq B$ and $n^{\prime} \leq L^{\prime}$ :

$$
\left(\frac{m}{\xi}-l^{\prime}\right) T^{(D)}+\frac{T_{s}^{(D)}}{\xi}+T_{g}^{(D)}-\tau_{n^{\prime}}^{(D)} \leq \theta^{(D)} \leq\left(\frac{m}{\xi}-l^{\prime}+1\right) T^{(D)}+T_{g}^{(D)}-\tau_{n^{\prime}}^{(D)}
$$

Therefore the interference can be written as

$$
\begin{aligned}
& I_{p}^{(D)}[m]=\frac{1}{\sqrt{T_{s}^{(D)} T_{s}^{(L)}}} \sum_{k^{\prime}=0}^{N^{(D)}-1} e^{-j 2 \pi \frac{k^{\prime}}{T_{s}^{(D)}} \theta^{(D)}}\left\{\sum_{n^{\prime} \in L_{b}} q_{n^{\prime}}^{(D)} e^{-j 2 \pi \frac{k^{\prime}}{T_{s}^{(D)} \tau_{n^{\prime}}^{(D)}}}\left(X_{k^{\prime}}^{(D)}\left[l^{\prime}\right]+X_{k^{\prime}}^{(D)}\left[l^{\prime}-1\right]\right) \times\right. \\
& \int_{\frac{m}{\xi} T^{(D)}}^{\frac{1}{\xi}\left(m T^{(D)}+T_{s}^{(D)}\right)} e^{j 2 \pi\left(\Delta f^{(D)}+\frac{k^{\prime}}{T_{s}^{(D)}}-\frac{p}{T_{s}^{(L)}}\right) t} d t+
\end{aligned}
$$

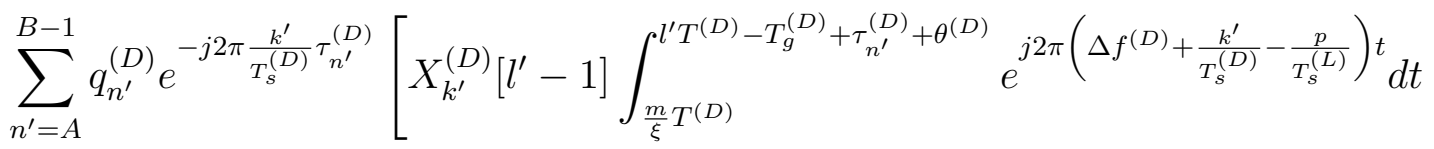

$$
\begin{aligned}
& \left.\left.+X_{k^{\prime}}^{(D)}\left[l^{\prime}\right] \int_{l^{\prime} T^{(D)}-T_{g}^{(D)}+\tau_{n^{\prime}}^{(D)}+\theta^{(D)}}^{\frac{1}{\xi}\left(m T^{(D)}+T_{s}^{(D)}\right)} e^{j 2 \pi\left(\Delta f^{(D)}+\frac{k^{\prime}}{T_{s}^{(D)}}-\frac{p}{T_{s}^{(L)}}\right) t} d t\right]\right\}
\end{aligned}
$$

with $L_{b}=\left\{1, . ., L^{\prime}\right\} \backslash\{A, \ldots, B-1\}$. Hence, the interference variance is obtained by averaging over $X_{k^{\prime}}^{(D)}, q_{n^{\prime}}^{(D)}$ then $\theta^{(D)}$. In practice, the maximum channel delay $\tau_{\max }^{(D)} \approx 5 \mu s<<T_{s}^{(L)}$. Consequently, we consider that the probability that $\theta^{(D)}$ is such that $\exists$ ( $A$ and $B$ ) leading to the situation previously described is negligible. Therefore, only two cases of the DVB interference over the LTE signal can be considered:

case 1: The time interval boundaries are described in (35), (36), (37) $\forall n^{\prime}$. In such a case, only one DVB symbol, i.e. $X_{k^{\prime}}^{(D)}\left[l^{\prime}\right]$ is interfering with the current LTE symbol, hence interference on the $p$-th subcarrier of the $m$-th OFDM symbol is given by:

$$
\begin{aligned}
& I_{p}^{(D)}[m]=\frac{1}{\sqrt{T_{s}^{(D)} T_{s}^{(L)}}} \sum_{k^{\prime}=0}^{N^{(D)}-1} X_{k^{\prime}}^{(D)}\left[l^{\prime}\right] \sum_{n^{\prime}=1}^{L^{\prime}} q_{n^{\prime}}^{(D)} e^{-j 2 \pi \frac{k^{\prime}}{T_{s}^{(D)}}\left(\tau_{n^{\prime}}^{(D)}+\theta^{(D)}\right)} \\
& \int_{m \frac{T^{(D)}}{\xi}}^{\frac{1}{\xi}\left(m T^{(D)}+T_{s}^{(D)}\right)} e^{j 2 \pi\left(\Delta f^{(D)}+\frac{k^{\prime}}{T_{s}^{(D)}}-\frac{p}{T_{s}^{(L)}}\right) t} d t \\
& =\sqrt{\frac{1}{\xi}} \sum_{k^{\prime}=0}^{N^{(D)}-1} X_{k^{\prime}}^{(D)}\left[l^{\prime}\right] e^{-j 2 \pi \frac{k^{\prime}}{T_{s}^{(D)}} \theta^{(D)}} Q_{k^{\prime}}^{(D)} \operatorname{sinc}\left(\pi\left(\frac{\Delta f^{(D)} T_{s}^{(D)}}{\xi}+\frac{k^{\prime}}{\xi}-p\right)\right) \times \\
& e^{j 2 \pi \frac{m T^{(D)}}{\xi}}\left(\Delta f^{(D)}+\frac{k^{\prime}}{T_{s}^{(D)}}-\frac{p}{T_{s}^{(L)}}\right) e^{j \pi\left(\frac{\Delta f^{(D)} T_{s}^{(D)}}{\xi}+\frac{k^{\prime}}{\xi}-p\right)}
\end{aligned}
$$


Averaging $I_{p}^{(D)}[m]$ over the symbols $X_{k^{\prime}}^{(D)}$ and the channel $q_{n^{\prime}}^{(D))_{3}}$, we obtain $\mathbb{E}_{X_{k^{\prime}}, q_{n^{\prime}}^{D}}^{(1)}\left[\left|I_{p}^{(D)}[m]\right|^{2}\right]$, defined in (8).

case 2: The time interval boundaries in (35) are such that $\forall n^{\prime}$ :

- For $m \frac{T^{(D)}}{\xi} \leq t \leq l^{\prime} T^{(D)}+\tau_{n^{\prime}}^{(D)}-T_{g}^{(D)}+\theta^{(D)} \rightarrow X_{k^{\prime}}^{(D)}\left[l^{\prime}-1\right]$ interferes.

- For $l^{\prime} T^{(D)}+\tau_{n^{\prime}}^{(D)}-T_{g}^{(D)}+\theta^{(D)} \leq t \leq \frac{1}{\xi}\left(m T^{(D)}+T_{s}^{(D)}\right) \rightarrow X_{k^{\prime}}^{(D)}\left[l^{\prime}\right]$ interferes.

which implies that:

$$
\left(\frac{m}{\xi}-l^{\prime}\right) T^{(D)}-\tau_{n^{\prime}}^{(D)}+T_{g}^{(D)}<\theta^{(D)}<\left(\frac{m}{\xi}-l^{\prime}\right) T^{(D)}-\tau_{n^{\prime}}^{(D)}+T_{g}^{(D)}+\frac{T_{s}^{(D)}}{\xi}
$$

In this case, two DVB symbols $X_{k^{\prime}}^{(D)}\left[l^{\prime}\right]$ and $X_{k^{\prime}}^{(D)}\left[l^{\prime}-1\right]$ interfere over the $p$-th subcarrier of the $m$-th LTE OFDM data block, and hence the broadcast interference is given by: Using $A\left(k^{\prime}\right)$ and $B\left(n^{\prime}, \theta^{(D)}\right)$, we obtain:

$$
\begin{aligned}
I_{p}^{(D)}[m]= & \frac{1}{\sqrt{T_{s}^{(D)} T_{s}^{(L)}}} \sum_{k^{\prime}=0}^{N^{(D)}-1} \frac{e^{-j 2 \pi \frac{k^{\prime}}{T_{s}^{(D)}} \theta^{(D)}}}{\pi A\left(k^{\prime}\right)} e^{j \pi A\left(k^{\prime}\right) \frac{m T^{(D)}}{\xi}} \sum_{n^{\prime}=1}^{n^{\prime}} q_{n^{\prime}}^{(D)} e^{-j 2 \pi \frac{k^{\prime}}{T_{s}^{(D)} \tau_{n^{\prime}}^{(D)}}} \times \\
& e^{j \pi A\left(k^{\prime}\right) B\left(n^{\prime}, \theta^{(D)}\right)}\left\{-X_{k^{\prime}}^{(D)}\left[l^{\prime}-1\right] \sin \left(\pi A\left(k^{\prime}\right)\left(\frac{m T^{(D)}}{\xi}-B\left(n^{\prime}, \theta^{(D)}\right)\right)\right)\right. \\
& \left.+X_{k^{\prime}}^{(D)}\left[l^{\prime}\right] e^{j \pi A\left(k^{\prime}\right) \frac{T_{s}^{(D)}}{\xi}} \sin \left(\pi A\left(k^{\prime}\right)\left(\frac{1}{\xi}\left(m T^{(D)}+T_{s}^{(D)}\right)-B\left(n^{\prime}, \theta^{(D)}\right)\right)\right)\right\}
\end{aligned}
$$

We aim at computing the average interference created by DVB system over LTE by averaging over symbols, channel coefficient and asynchronism ${ }^{4}$. The DVB interference averaged over the symbols then over the channel is given by:

$$
\begin{aligned}
\mathbb{E}_{X_{k^{\prime}}, q_{n^{\prime}}^{D}}^{(2)}\left[\left|I_{p}^{(D)}[m]\right|^{2}\right]= & \frac{1}{T_{s}^{(D)} T_{s}^{(L)}} \sum_{k^{\prime}=0}^{N^{(D)}-1} \frac{1}{\pi^{2} A\left(k^{\prime}\right)^{2}} \\
& \left\{\sum_{n^{\prime}=0}^{L^{\prime}}\left|\Omega_{n^{\prime}}^{(D)}\right|^{2} \sin ^{2}\left(\pi A\left(k^{\prime}\right)\left(\frac{1}{\xi}\left(m T^{(D)}+T_{s}^{(D)}\right)-B\left(n^{\prime}, \theta^{(D)}\right)\right)\right)+\right. \\
& \left.\sum_{n^{\prime}=0}^{L^{\prime}}\left|\Omega_{n^{\prime}}^{(D)}\right|^{2} \sin ^{2}\left(\pi A\left(k^{\prime}\right)\left(\frac{m T^{(D)}}{\xi}-B\left(n^{\prime}, \theta^{(D)}\right)\right)\right)\right\}
\end{aligned}
$$

where the mean of the double products give null value because of the independence of the symbols $X_{k^{\prime}}\left[l^{\prime}\right]$ and $x_{k^{\prime}}\left[l^{\prime}-1\right]$ and the coefficients $q_{n^{\prime}}^{D}$ are independent from each other. Taking

${ }^{3}$ Delays are considered as deterministic values.

${ }^{4}$ The averaging order is not important, however this one leads to the simplest derivations. 
into account both cases of $\theta^{(D)}$, the previous interference expression should be averaged over $\theta^{(D)}$ which is assumed to be uniformly distributed over $\left[0, T^{(D)}[\right.$ and its probability density function (pdf) is given by:

$$
f_{\theta^{(D)}}=\left\{\begin{array}{cc}
\frac{1}{T^{(D)}} & \forall \theta^{(D)} \in\left[0, T^{(D)}[\right. \\
0 & \text { elsewhere }
\end{array}\right.
$$

The variation domain of $\theta^{(D)}$ leading to case 2 is such as $\theta^{D} \in\left[0, \frac{T_{s}^{(D)}}{\xi}\right]$. Therefore, the DVB interference over the LTE signal in case 2 averaged by $\theta^{(D)}$ is given by:

$$
\mathbb{E}_{x_{k^{\prime}}, q_{n^{\prime}}^{(D)}, \theta^{(D)}}^{(2)}\left[\left|I_{p}^{(D)}[m]\right|^{2}\right]=\frac{1}{T_{s}^{(D)} / \xi} \int_{0}^{T_{s}^{(D)} / \xi} \mathbb{E}_{X_{k^{\prime}}, q_{n^{\prime}}^{(D)}}^{(2)}\left[\left|I_{p}^{(D)}[m]\right|^{2}\right] d \theta^{(D)}
$$

After trigonometric considerations and tedious calculation, the interference variance for case 2 is obtained in (9). For case 1, the interference variance expression depends on $\theta^{(D)}$ implicitly. The probability that case 1 occurs is equal to the probability that $\theta^{(D)}$ belongs to $\left[T_{s}^{(D)} / \xi, T^{(D)}[\right.$. Therefore we obtain (7).

\section{APPENDIX B}

\section{ProOF OF THEOREM III. 2}

Before sampling, the LTE received signal at DR can be expressed as:

$$
\begin{array}{r}
r^{(L)}(t)=\frac{e^{j 2 \pi \Delta f^{(L)} t}}{\sqrt{T_{s}^{(L)}}} \sum_{l \in \mathbb{Z}} \sum_{k=0}^{N^{(L)}-1} \sum_{n=1}^{L} X_{k}^{(L)}[l] q_{n}^{(L)} e^{j 2 \pi \frac{k}{T_{s}^{(L)}}\left(t-\tau_{l}^{(L)}-\theta^{(L)}\right)} \\
\Pi\left(\frac{t-\tau_{l}^{(L)}-l T^{(L)}+T_{g}^{(L)}-\theta^{(L)}}{T^{(L)}}\right)
\end{array}
$$

where $\Delta f^{(L)}$ is the LTE frequency shift w.r.t. DVB. DVB receiver decomposes the interfering LTE signal according to its own basis. Hence, the interference received on the $p^{\prime}-$ th subcarrier and during the $m^{\prime}-$ th LTE OFDM data block is given by

$$
\begin{aligned}
I_{p^{\prime}}^{(L)}\left[m^{\prime}\right]= & \int_{\mathbb{R}} r^{(L)}(t) \phi_{p^{\prime}, m^{\prime}}^{(D)}(t) d t \\
= & \frac{1}{\sqrt{T_{s}^{(D)} T_{s}^{(L)}}} \int_{t} e^{j 2 \pi \Delta f^{(L)} t} \sum_{l \in \mathbb{Z}} \sum_{k=0}^{N^{(L)}-1} \sum_{n=1}^{L} X_{k}^{(L)}[l] q_{n}^{(L)} e^{j 2 \pi \frac{k}{T_{s}^{(L)}\left(t-\tau_{l}^{(L)}-\theta^{(L)}\right)} e^{-j 2 \pi \frac{p^{\prime}}{T_{s}^{(D)} t}}} \\
& \Pi\left(\frac{t-m^{\prime} T^{(D)}}{T_{s}^{(D)}}\right) \Pi\left(\frac{t-\tau_{l}^{(L)}-l T^{(L)}+T_{g}^{(L)}-\theta^{(L)}}{T^{(L)}}\right)
\end{aligned}
$$


where

$$
\begin{aligned}
& \Pi\left(\frac{t-m^{\prime} T^{(D)}}{T_{s}^{(D)}}\right)=1 \text { iff }\left\{\begin{array}{l}
m^{\prime} T^{(D)} \leq t \leq m^{\prime} T^{(D)}+T_{s}^{(D)} \\
m^{\prime} \xi T^{(L)} \leq t \leq \xi\left(m^{\prime} T^{(L)}+T_{s}^{(L)}\right)
\end{array}\right. \\
& \Pi\left(\frac{t-\tau_{n}^{(L)}-l T^{(L)}+T_{g}^{(L)}-\theta^{(L)}}{T^{(L)}}\right)=1 \quad \text { iff } l T^{(L)}+\tau_{n}^{(L)}-T_{g}^{(L)}+\theta^{(L)} \leq t \\
& \leq(l+1) T^{(L)}+\tau_{n}^{(L)}-T_{g}^{(L)}+\theta^{(L)}
\end{aligned}
$$

with $\theta^{(L)} \sim \mathcal{U}\left(\left[0, T^{(L)}\right]\right)$. Moreover, the DVB OFDM symbol duration is larger than the LTE OFDM symbol duration and is not a multiple of it. The numbers of LTE symbols interfering on DVB symbol is $\xi^{\prime} \triangleq \frac{T_{s}^{(D)}}{T^{(L)}}=\xi \frac{T_{s}^{(D)}}{T^{(D)}}$. Therefore, $\left\lfloor\xi^{\prime}\right\rfloor$ LTE symbols interfere entirely over DVB symbol: $X_{k}^{(L)}[l], X_{k}^{(L)}[l+1], X_{k}^{(L)}[l+2] \ldots, X_{k}^{(L)}\left[\left\lfloor\xi^{\prime}\right\rfloor-1\right]$. Moreover, the $(l-1)$-th and $\left\lfloor\xi^{\prime}\right\rfloor$-th LTE symbols interfere partially respectively at the beginning and the end of DVB symbol. Applying the same calculation steps than those presented in the case of DVB interfering signal over LTE signal, the received interference over the $p^{\prime}$-th DVB subcarrier and the $m^{\prime}$-th data block can be written as:

$$
\begin{aligned}
& I_{p^{\prime}}^{(L)}\left[m^{\prime}\right]=\frac{1}{\sqrt{T_{s}^{(D)} T_{s}^{(L)}}} \sum_{k=0}^{N^{(L)}-1} e^{-j 2 \pi \frac{k}{T_{s}^{(L)}} \theta^{(L)}} \times \\
& \left\{-X_{k}^{(L)}[l-1] e^{j \pi C(k) m \xi T^{(L)}} \sum_{n=1}^{L} q_{n}^{(L)} e^{-j \pi\left(C(k) G\left(n, \theta^{(L)}\right)+2 \frac{k}{T_{s}^{(L)}} \tau_{n}^{(L)}\right)}\right. \\
& \frac{\sin \left(\pi C(k)\left(m \xi T^{(L)}-G\left(n, \theta^{(L)}\right)\right)\right)}{\pi C(k)} \\
& +\left(T^{(L)} \sin \left(\pi C(k) T^{(L)}\right) e^{j 2 \pi C(k) T^{(L)}} \sum_{b=0}^{\left\lfloor\xi^{\prime}\right\rfloor-1} X_{k}^{(L)}[l+b] e^{j 2 \pi C(k) b T^{(L)}}\right) \\
& \sum_{n=1}^{L} q_{n}^{(L)} e^{-j 2 \pi\left(C(k) G\left(n, \theta^{(L)}\right)-\frac{k}{T_{s}^{L}} \tau_{n}^{(L)}\right)} \\
& \left.+X_{k}^{(L)}\left[\left\lfloor\xi^{\prime}\right\rfloor\right] e^{j \pi C(k)\left(\left\lfloor\xi^{\prime}\right\rfloor T^{(L)}+\xi m T^{(L)}+\xi T_{s}^{(L)}\right)} \sum_{n=1}^{L} q_{n}^{(L)} e^{-j \pi\left(C(k) G\left(n, \theta^{(L)}\right)+2 \frac{k}{T_{s}^{(L)}} \tau_{n}^{(L)}\right.}\right) \\
& \left.\frac{\sin \left(\pi C(k)\left(\xi\left(m T^{(L)}+T_{s}^{(L)}\right)-\left\lfloor\xi^{\prime}\right\rfloor T^{(L)}-G\left(n, \theta^{(L)}\right)\right)\right)}{\pi C(k)}\right\}
\end{aligned}
$$


where $C(k)=\Delta f^{(L)}+\frac{k}{T_{s}^{(L)}}-\frac{p^{\prime}}{T_{s}^{(D)}}$ and $G\left(n, \theta^{(L)}\right)=l T^{(L)}-T_{g}^{(L)}+\tau_{n}^{(L)}+\theta^{(L)}$. Averaging over the symbols, channel and asynchronism, we obtain (13).

\section{REFERENCES}

[1] CEPT, "The identification of common and minimal (least restrictive) technical conditions for $790-862 \mathrm{MHz}$ for the digital dividend in the European Union," Electronic Communications Committee (ECC) within the European Conference of Postal and Telecommunications Administrations (CEPT), Tech. Rep., October 2009.

[2] M. M. Anis, X. Lagrange, and R. Pyndiah, “A simple model for DVB and LTE cooperation,” in 2013 IEEE International Symposium on Broadband Multimedia Systems and Broadcasting (BMSB), London, 2013, pp. 1-6.

[3] A. A. Razzac, S. E. Elayoubi, T. Chahed, and B. E. Hassan, "Comparison of LTE eMBMS and DVB-NGH mobile TV solutions from an energy consumption perspective," in Workshop on End-to-End Green Cellular Networks, IEEE 24th International Symposium on Personal, Indoor and Mobile Radio communications, 2013, pp. 1-8.

[4] A. A. Vopilov and S. A. Kravchuk, "Use of modern wireless technologies in combined LTE/DVB-NGH systems," in Microwave and Telecommunication Technology (CriMiCo), 2013 23rd International Crimean Conference, Sevastopol, 2013, pp. 285-286.

[5] A. Aloisi, M. Celidonio, L. Pulcini, and A. Rufini, "A simulation study of broadband mobile systems interference on DVB-T systems operating in the UHF frequency band," in Future Network Mobile Summit (FutureNetw), 2011, Warsaw, 2011, pp. 1-8.

[6] W. Li, J. Chen, H. Long, and B. Wu, "Performance and analysis on LTE system under adjacent channel interference of broadcasting system," in Computer and Information Technology (CIT), 2012 IEEE 12th International Conference on, Oct 2012, pp. 290-294.

[7] L. Polak, O. Kaller, L. Klozar, and J. Prokopec, "Exploring and measuring the co-existence between LTE and DVB-T2Lite services," in Telecommunications and Signal Processing (TSP), 2013 36th International Conference on, July 2013, pp. 316-320.

[8] L. Polak, O. Kaller, L. Klozar, J. Sebesta, and T. Kratochvil, "Mobile communication networks and digital television broadcasting systems in the same frequency bands: Advanced co-existence scenarios," Radioengineering, vol. 23, no. 1, pp. 375-386, April 2014.

[9] L. Polak, L. Klozar, O. Kaller, J. Sebesta, M. Slanina, and T. Kratochvil, "Study of coexistence between indoor LTE femtocell and outdoor-to-indoor DVB-T2-lite reception in a shared frequency band," EURASIP Journal on Wireless Communications and Networking, no. 1, p. 114, 2015.

[10] J. Kristel, L. Polak, and T. Kratochvil, "Co-channel coexistence between DVB-T/H and LTE standards in a shared frequency band," in Radioelektronika (RADIOELEKTRONIKA), 2015 25th International Conference, Pardubice, 2015, pp. 184-190.

[11] M. Ferrante, G. Fusco, E. Restuccia, M. Celidonio, P. G. Masullo, and L. Pulcini, "Experimental results on the coexistence of TV broadcasting service with LTE mobile systems in the $800 \mathrm{MHz}$ band," in Euro Med Telco Conference (EMTC), 2014, Naples, 2014, pp. 1-6.

[12] A. Aloisi, M. Celidonio, L. Pulcini, and A. Rufini, "Experimental study on Protection Distances between LTE and DVB-T stations operating in adjacent UHF frequency bands," in Wireless Telecommunications Symposium (WTS) 2011, 13-15 April 2011, pp. 1-7.

[13] _ _ "A simulation study of broadband mobile systems interference on DVB-T systems operating in the UHF frequency band," in Future Network \& Mobile Summit (FutureNetw), 2011, 15-17 June 2011, pp. 1-8. 
[14] M. Celidonio, P. G. Masullo, L. Pulcini, M. V. Die, G. Fusco, and M. Ferrante, "LTE services over the $800 \mathrm{MHz}$ band: Interference effects on DVB-T channels caused by the uplink signal," in 2015 AEIT International Annual Conference (AEIT), Naples, 2015, pp. 1-6.

[15] M. Celidonio, P. G. Masullo, L. Pulcini, and M. Vaser, "Measured interference of LTE uplink signals on DVB-T channels," Journal of telecommunications and information technology, pp. 74-85, 42015.

[16] R. Liyanapathirana, U. Gunawardana, P. Wijesinghe, and S. Biyanwilage, "Rf interference to DVB-T reception from UMTS/LTE systems in adjacent bands," in TENCON Spring Conference, 2013 IEEE, Sydney, NSW, 2013, pp. 398-402.

[17] S. Sam, S. Biyanwilage, U. Gunawardana, and R. Liyanapathirana, "Rf interference to DVB-T reception from multiple LTE devices in adjacent bands," in World of Wireless, Mobile and Multimedia Networks (WoWMoM), 2014 IEEE 15th International Symposium on a, Sydney, NSW, 2014, pp. 1-3.

[18] S. Basnet, U. Gunawardana, S. Biyanwilage, and R. Liyanapathirana, "Interference analysis in digital TV reception with LTE systems in adjacent bands in australian context," in Telecommunication Networks and Applications Conference (ATNAC), 2014 Australasian, Southbank, VIC, 2014, pp. 82-86.

[19] A. Tekovic, G. Šimac, and K. Šakić, "LTE downlink system performance measurement with intersystem interference caused by DVB-T signal," in ELMAR, 2012 Proceedings, Zadar, 2012, pp. 255-258.

[20] J. Ribadeneira-Ramírez, G. Martínez, D. Gómez-Barquero, and N. Cardona, "Interference analysis between digital terrestrial television (dtt) and 4g LTE mobile networks in the digital dividend bands," IEEE Transactions on Broadcasting, vol. 62, no. 1, pp. 24-34, March 2016.

[21] G. Baruffa, M. Femminella, F. Mariani, and G. Reali, "Protection ratio and antenna separation for DVB-T/LTE coexistence issues," IEEE Communications Letters, vol. 17, no. 8, pp. 1588-1591, August 2013.

[22] G. Martínez-Pinzón, N. Cardona, C. Garcia-Pardo, A. Fornés-Leal, and J. A. Ribadeneira-Ramírez, "Spectrum sharing for LTE-A and DTT: Field trials of an indoor LTE-A femtocell in DVB-T2 service area," IEEE Transactions on Broadcasting, vol. 62, no. 3, pp. 552-561, Sept 2016.

[23] A. Tekovic, D. Bonefacic, R. Nad, J. Pidanic, and Z. Nemec, "On performance of filter used for interference mitigation between LTE and DVB-T networks in digital dividend spectrum," in 2016 International Symposium ELMAR, Sept 2016, pp. 17-20.

[24] L. Polak, O. Kaller, L. Klozar, M. Slanina, J. Sebesta, and T. Kratochvil, "Coexistence between DVB-T/T2 and LTE standards in common frequency bands," Wireless Personal Communications, vol. 88, no. 3, pp. 669-684, Jun 2016. [Online]. Available: https://doi.org/10.1007/s11277-016-3191-2

[25] H. Bawab, P. Mary, J. F. Hélard, Y. Nasser, and O. Bazzi, "Global Ergodic Capacity Closed-Form Expression of Coexisting DVB-LTE-Like Systems,” in Vehicular Technology Conference (VTC Spring'14), 2014 IEEE 79th, 2014, pp. 1-5.

[26] — - "Ergodic capacity optimization in coexisting DVB-LTE-like systems," in Ultra Modern Telecommunications and Control Systems and Workshops (ICUMT), 2014 6th International Congress on, 6-8 Oct. 2014, pp. 54,59.

[27] F. Haider, C. X. Wang, H. Haas, E. Hepsaydir, X. Ge, and D. Yuan, "Spectral and energy efficiency analysis for cognitive radio networks," IEEE Transactions on Wireless Communications, vol. 14, no. 6, pp. 2969-2980, June 2015.

[28] P. Mary, J. M. Gorce, G. Villemaud, M. Dohler, and M. Arndt, "Reduced Complexity MUD-MLSE Receiver for PartiallyOverlapping WLAN-Like Interference,” in 2007 IEEE 65th Vehicular Technology Conference - VTC2007-Spring, April 2007, pp. 1876-1880.

[29] —_ "Performance analysis of mitigated asynchronous spectrally-overlapping WLAN interference," in 2007 IEEE Wireless Communications and Networking Conference, March 2007, pp. 2097-2102.

[30] L. Zeng and S. McGrath, "Joint spectrum sensing and power allocation algorithm for spectrum efficiency optimization in 
ultra wideband cognitive radio networks," in Vehicular Technology Conference (VTC Fall), 2012 IEEE, Quebec City, QC, 2012, pp. 1-5.

[31] Y. Wu, Y. Sun, Y. Ji, J. Mao, and Y. Liu, "A joint channel allocation and power control scheme for interference mitigation in high-density wlans," in Communication Technology (ICCT), 2013 15th IEEE International Conference on, Guilin, 2013, pp. 98-103.

[32] M. De Mari, E. C. Strinati, and M. Debbah, "Two-regimes interference classifier: An interference-aware resource allocation algorithm," in 2014 IEEE Wireless Communications and Networking Conference (WCNC), Istanbul, 2014, 2014, pp. 792797.

[33] S. Paranche Damodaran and M. Pazha, "Analysis of interference modeling and coexistence for next-generation LTE cellular systems," in Communications and Signal Processing (ICCSP), 2015 International Conference on, Melmaruvathur, 2015, pp. $1608-1611$.

[34] S. Sorooshyari, C. W. Tan, and M. Chiang, "Power control for cognitive radio networks: Axioms, algorithms, and analysis," IEEE/ACM Transactions on Networking, vol. 30, no. 3, pp. 878-891, June 2012.

[35] D. Das and S. Das, "Interference-aware power allocation in soft decision fusion (SDF) based cooperative spectrum sensing," in 2014 Annual IEEE India Conference (INDICON), Pune, 2014, pp. 1-6. 